Research Article

\title{
Stability and Bifurcation Analysis of a Discrete Singular Bioeconomic System
}

\author{
Qamar Din $\mathbb{D}^{1}{ }^{1}$ A. M. Yousef, ${ }^{2}$ and A. A. Elsadany ${ }^{3,4}$ \\ ${ }^{1}$ Department of Mathematics, University of Poonch Rawalakot, Rawalakot 12350, Pakistan \\ ${ }^{2}$ Mathematics Department, Faculty of Science, South Valley University, Qena, Egypt \\ ${ }^{3}$ Mathematics Department, College of Science and Humanities Studies in Al-Kharj, Prince Sattam Bin Abdulaziz University, \\ Al-Kharj 11942, Saudi Arabia \\ ${ }^{4}$ Department of Basic Science, Faculty of Computers and Informatics, Suez Canal University, Ismailia 41522, Egypt
}

Correspondence should be addressed to Qamar Din; qamar.sms@gmail.com

Received 23 December 2020; Accepted 5 July 2021; Published 13 July 2021

Academic Editor: Douglas Anderson

Copyright (C) 2021 Qamar Din et al. This is an open access article distributed under the Creative Commons Attribution License, which permits unrestricted use, distribution, and reproduction in any medium, provided the original work is properly cited.

The main concern of this paper is to discuss stability and bifurcation analysis for a class of discrete predator-prey interaction with Holling type II functional response and harvesting effort. Firstly, we establish a discrete singular bioeconomic system, which is based on the discretization of a system of differential algebraic equations. It is shown that the discretized system exhibits much richer dynamical behaviors than its corresponding continuous counterpart. Our investigation reveals that, in the discretized system, two types of bifurcations (i.e., period-doubling and Neimark-Sacker bifurcations) can be studied; however, the dynamics of the continuous model includes only Hopf bifurcation. Moreover, the state delayed feedback control method is implemented for controlling the chaotic behavior of the bioeconomic model. Numerical simulations are presented to illustrate the theoretical analysis. The maximal Lyapunov exponents (MLE) are computed numerically to ensure further dynamical behaviors and complexity of the model.

\section{Introduction}

Bioeconomics is linked closely to the early development of ideas in fisheries economics due to the pioneering work of Canadian economists Gordon [1] and Anthony Scott (in 1955). Their basic theories used recent developments in modeling of biological fisheries, initially the contributions made by Schaefer in 1954 and 1957 on introducing a systematic connection between fishing mechanism and growth of biological type through the implementation of mathematical modeling verified by experimental studies, and also associated itself to resource protection, ecology, and the environment [2]. These concepts were developed from the multifishing science environment in Canada at that time. Modeling and fishing science developed rapidly during an innovative and productive period, especially among Canadian fishing researchers of various disciplines. Fishing mortality and population modeling were launched for economists, and novel interdisciplinary methods of modeling became obtainable for the economists, which made it feasible to measure the economical and biological impacts of various fisheries management decisions and fishing activities. Modern bioeconomics related to fisheries science can furnish perception into developing methods to deal with the overexploitation and complexities of overcapacity in marine fisheries where most are affected by lack of solid governance, changing coastal ecosystem dynamics, and natural fluctuations [3].

Moreover, Gordon in [1] suggested economic theory keeping in view the common property of resource, which was based on the effect of the harvest effort on an ecosystem by taking into account an economic perspective, assuming that $x(t)$ and $e(t)$ denote the density of harvested population and the harvest effort in an ecosystem, respectively; then the total cost is equal to $c e(t)$, and the total revenue is equal to $p e(t) x(t)$, where $c$ denotes the cost of harvest effort, and $p$ is 
used for the unit price of harvested population. Then, the economic interest $\mu$ for the harvest effort by the harvested population is given by

$$
\mu=e(t)(p x(t)-c) .
$$

Taking into account Gordon [1] theory, Zhang et al. [4] studied a class of bioeconomic system with implementation of theory for singular systems. Their study was consisted of bifurcation analysis and chaos control for the proposed bioeconomic model. Later on, Liu et al. [5] reported stability, bifurcation analysis, and state feedback control for a class of predator-prey interaction with harvest effort on predator and stage structure for prey. Chakraborty et al. [6] studied a bioeconomic system with implementation of theory of differential algebraic equations. They investigated stability, Hopf bifurcation, and state feedback control for a class of predator-prey interaction with time-delayed effect. Zhang et al. [7] investigated a singular bioeconomic model for preypredator interaction with diffusion and time delay. Zhang et al. [8] carried out comprehensive study related to theory, applications, complexity, and control of singular bioeconomic systems. Zhang et al. [9] explored the Hopf bifurcation for a predator-prey type bioeconomic system with two delays and predator harvesting. Meng and Zhang [10] discussed the qualitative behavior of a delayed singular bioeconomic predator-prey model without and with stochastic fluctuation. Liu et al. [11] analyzed the local dynamics and Hopf bifurcation for a biological economic model with Holling type II functional response and harvesting effort on prey. Liu et al. [12] proposed a singular fishery model for a class of prey-predator interaction with gestation delay for predator and maturation delay for prey. Liu et al. [13] formulated and discussed a singular predatorprey model by implementing commercial harvesting on predator with gestation delay for predator and maturation delay for prey. In [14], Li et al. studied a singular bioeconomic predator-prey system with Holling type II functional response and nonlinear harvesting on prey. Meng and $\mathrm{Wu}$ [15] discussed a singular prey-predator system with two delays, nonlinear predator harvesting and Beddington-DeAngelis functional response. Babaei and Shafiee [16] reported stability analysis, bifurcation, chaotic behavior, and control for a singular bioeconomic model of prey-predator interaction governed by an algebraic equation and 3-dimensional differential equations.

In case of mathematical modeling of predator-prey interaction, the research concerning interspecific interactions has been numerously based on continuous predator-prey systems of two variables. On the other hand, particular species, covering several classes of insects and seasonal plants, have nonoverlapping generations successively, and consequently, their population undergoes in discrete time steps. Populations with nonoverlapping generations can be modeled suitably with difference equations, otherwise known as iterative maps or discrete dynamical systems. Several authors have shown that nonoverlapping generations governed by iterative maps reveal complex and chaotic behavior, and the dynamics in such cases may yield a much richer set of patterns than those examined in continuoustime systems (cf. [17-24]). Furthermore, discrete-time models also have been used for rich dynamics of bioeconomic systems for some classes of predator-prey interaction. For example, in [25], the authors analyzed complex dynamics of a discrete-time bioeconomic system for predator-prey interaction with the implementation of Euler approximations. $\mathrm{Wu}$ and Chen [26] implemented the Poincaré scheme for discretization of a singular bioeconomic model and they analyzed period-doubling bifurcation, Neimark-Sacker bifurcation, and stability behavior. Liu et al. [27] studied the chaotic behavior of a discrete singular system related to the bioeconomic model of the prey-predator type.

Taking into account predator interaction with logistic growth and Holling type II functional response for prey population, we have the following system [28]:

$$
\left\{\begin{array}{l}
\frac{\mathrm{d} x}{\mathrm{~d} t}=x\left(d-k x-\frac{y}{a+x}\right) \\
\frac{\mathrm{d} y}{\mathrm{~d} t}=y\left(\frac{b x}{a+x}-r\right)
\end{array}\right.
$$

where $x=x(t)$ and $y=y(t)$ denote state variables for the densities of prey and predator at time $t$, respectively. Moreover, $d$ is the intrinsic growth rate of prey, $r$ represents the natural death rate of the predator, $a$ is used for half capturing saturation constant, and $b$ represents the maximal growth rate of the predator. Furthermore, $d / k$ denotes the environmental carrying capacity for the prey population.

Keeping in view (1) and (2), we obtain the following predator-prey biological economic model with Holling type II functional response with harvest effort:

$$
\left\{\begin{array}{l}
\frac{\mathrm{d} x}{\mathrm{~d} t}=x\left(d-k x-\frac{y}{a+x}-e\right) \\
\frac{\mathrm{d} y}{\mathrm{~d} t}=y\left(\frac{b x}{a+x}-r\right) \\
\mu=e(p x-c)
\end{array}\right.
$$

Applying the forward Euler scheme to system (3), we obtain the discrete-time predator-prey biological economic model with Holling type II functional response as follows:

$$
\left\{\begin{array}{l}
x_{n+1}=x_{n}+h x_{n}\left(d-k x_{n}-\frac{y_{n}}{a+x_{n}}-e_{n}\right), \\
y_{n+1}=y_{n}+h y_{n}\left(\frac{b x_{n}}{a+x_{n}}-r\right), \\
\mu=e_{n}\left(p x_{n}-c\right),
\end{array}\right.
$$

where $h$ is the integral step size for the Euler approximation. In this paper, we discuss some dynamical aspects of the discrete singular model (4). For this, the first existence of 
interior (positive) fixed point and local dynamics of system (4) about biologically feasible equilibrium are carried out. Secondly, it is proved that system (4) undergoes perioddoubling bifurcation and Neimark-Sacker bifurcation by varying the economic profit $\mu$ as the bifurcation parameter. Thirdly, a state delayed feedback control strategy is applied to avoid bifurcating and chaotic behavior of bioeconomic model (4). At the end, numerical examples are presented for verification and illustration of our theoretical discussion.

\section{Fixed Points and Stability Analysis}

In order to study the qualitative behavior of the solutions of the nonlinear model (4), we study the existence of fixed points and their stability properties. From system (4), we can see that there exists a fixed point $X_{0}:=\left(x_{0}, y_{0}, e_{0}\right)$ in $R_{3}^{+}$if and only if $X_{0}$ is a solution of the following equations:

$$
\left\{\begin{array}{l}
x=x+h x\left(d-k x-\frac{y}{a+x}-e\right) \\
y=y+h y\left(-r+\frac{b x}{a+x}\right) \\
\mu=e(p x-c) .
\end{array}\right.
$$

Through a simple calculation, we obtain

$$
X_{0}=\left(x_{0}, y_{0}, e_{0}\right)=\left(\frac{a r}{(b-r)},\left(a+x_{0}\right)\left(d-k x_{0}-e_{0}\right), \frac{\mu}{\left(p x_{0}-c\right)}\right) \text {. }
$$

For biological considerations, we focus on the dynamics of the positive fixed point of the system (4). Thus, throughout the paper, we assume the conditions for the existence of a unique positive fixed point of system (4) as follows:

$$
\begin{aligned}
& b>r, \\
& b c<r(a p+c), \\
& \frac{b d-r(a k+d)}{(b-r)^{2}}-\frac{\mu}{r(a p+c)-b c}>0 .
\end{aligned}
$$

In $\mu k$-plane, the existence of a unique positive fixed point of system (4) is depicted in Figure 1.

The generalized Jacobian matrix $J\left(x_{0}, y_{0}, e_{0}\right)$ of system (4) about interior (positive) fixed point $\left(x_{0}, y_{0}, e_{0}\right)$ is computed as follows:

$$
J\left(x_{0}, y_{0}, e_{0}\right)=\left(\begin{array}{ccc}
1-h k x_{0}+\frac{h x_{0} y_{0}}{\left(a+x_{0}\right)^{2}} & -\frac{h x_{0}}{a+x_{0}} & -h x_{0} \\
\frac{a b h y_{0}}{\left(a+x_{0}\right)^{2}} & 1 & 0 \\
e_{0} p & 0 & p x_{0}-c
\end{array}\right) \text {. }
$$

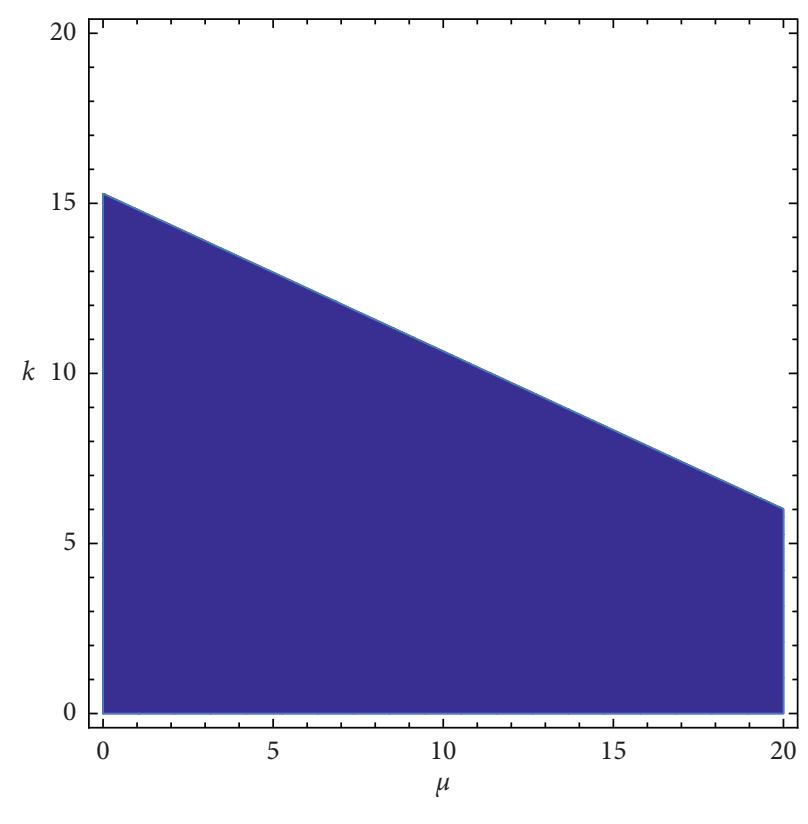

Coexistence region

FIGURE 1: Coexistence region for system (4) with $a=6.76, b=9.4$, $c=1.58, r=0.74, p=9.2$, and $d=8.83$.

Then, it is easy to see that the generalized characteristic equation of the Jacobian matrix $J\left(x_{0}, y_{0}, e_{0}\right)$ can be written as

$$
\operatorname{det}\left(\begin{array}{ccc}
1-h k x_{0}+\frac{h x_{0} y_{0}}{\left(a+x_{0}\right)^{2}}-\lambda & -\frac{h x_{0}}{a+x_{0}} & -h x_{0} \\
\frac{a b h y_{0}}{\left(a+x_{0}\right)^{2}} & 1-\lambda & 0 \\
e_{0} p & 0 & p x_{0}-c
\end{array}\right)=0 \text {, }
$$

which on simplification yields

$$
\lambda^{2}+P \lambda+Q=0,
$$

where

$$
\begin{aligned}
P & =h k x_{0}-\frac{h x_{0} y_{0}}{\left(a+x_{0}\right)^{2}}-\frac{h e_{0} p x_{0}}{p x_{0}-c}-2 \\
& =\frac{1}{((\operatorname{arp} /(b-r))-c)^{2}}\left[R_{1} \mu-\left(S_{1}+2\right)\left(\frac{\operatorname{arp}}{b-r}-c\right)^{2}\right]=H-Q-1, \\
Q & =1-h k x_{0}+\frac{h x_{0} y_{0}}{\left(a+x_{0}\right)^{2}}+\frac{h e_{0} p x_{0}}{p x_{0}-c}+\frac{a b h^{2} x_{0} y_{0}}{\left(a+x_{0}\right)^{3}} \\
& =\frac{1}{((\operatorname{arp} /(b-r))-c)^{2}}\left[R_{2} \mu-\left(S_{2}-1\right)\left(\frac{\operatorname{arp}}{b-r}-c\right)^{2}\right],
\end{aligned}
$$

where 


$$
\begin{aligned}
& H=\frac{a b x_{0} y_{0}}{\left(a+x_{0}\right)^{3}}>0, \\
& R_{1}=-\frac{h r}{b}(a p+c), \\
& S_{1}=-\frac{h r}{b}\left(d-\frac{a k(b+r)}{b-r}\right), \\
& R_{2}=\frac{h r}{b}[(a p+c)(1-h r)+h c b], \\
& S_{2}=\frac{h r}{b}\left[d-\frac{a k(b+r)}{b-r}+h d(b-r)-h a r k\right] .
\end{aligned}
$$

Let $F(\lambda)=\lambda^{2}+P \lambda+Q$, then

$$
\begin{aligned}
F(1)= & 1+P+Q=H>0, \\
F(-1)= & 1-P+Q=\frac{2}{((\operatorname{arp} /(b-r))-c)^{2}} \\
& {\left[R_{3} \mu-\left(S_{3}-2\right)\left(\frac{\operatorname{arp}}{b-r}-c\right)^{2}\right], }
\end{aligned}
$$

where

$$
\begin{aligned}
& R_{3}=\frac{h r}{b}\left[(a p+c)\left(1-\frac{h r}{2}\right)+\frac{h c b}{2}\right], \\
& S_{3}=\frac{h r}{b}\left[d-\frac{a k(b+r)}{b-r}+\frac{h d(b-r)}{2}-\frac{h a r k}{2}\right] .
\end{aligned}
$$

In order to discuss the stability of the fixed point of $\left(x_{0}, y_{0}, e_{0}\right)$, we need the following lemma.

Lemma 1 (see [23]). Consider $S(\zeta)=\zeta^{2}+P \zeta+Q$. Moreover, assuming that $S(1)>0$ and $\zeta_{1}$ and $\zeta_{2}$ are two roots of $S(\zeta)=0$, then the following hold true:

(i) $\left|\zeta_{1}\right|<1$ and $\left|\zeta_{2}\right|<1$ if and only if $S(-1)>0$, and $S(0)=Q<1$

(ii) $\left|\zeta_{1}\right|<1$ and $\left|\zeta_{2}\right|>1$ (or $\left|\zeta_{1}\right|>1$ and $\left|\zeta_{2}\right|<1$ ) if and only if $S(-1)<0$

(iii) $\left|\zeta_{1}\right|>1$ and $\left|\zeta_{2}\right|>1$ if and only if $S(-1)>0$ and $S(0)>1$

(iv) $\zeta_{1}=-1$ and $\left|\zeta_{2}\right| \neq 1$ if and only if $S(-1)=0$ and $P \neq 0,2$

(v) $\zeta_{1}$ and $\zeta_{2}$ are complex and $\left|\zeta_{1}\right|=1$ and $\left|\zeta_{2}\right|=1$ if and only if $P^{2}-4 Q<0$ and $Q=1$

Assume that $\zeta_{1}$ and $\zeta_{2}$ are roots for the characteristic equation of the variational matrix $J\left(x_{0}, y_{0}, e_{0}\right)$ about interior (positive) fixed point $\left(x_{0}, y_{0}, e_{0}\right)$ which are known as eigenvalues for the equilibrium point $\left(x_{0}, y_{0}, e_{0}\right)$. Taking into account the topological types related to the fixed point $\left(x_{0}, y_{0}, e_{0}\right)$ of system (4), we say that the fixed point $\left(x_{0}, y_{0}, e_{0}\right)$ is a sink (asymptotically stable) if $\left|\zeta_{1}\right|<1$ and $\left|\zeta_{2}\right|<1 ;\left(x_{0}, y_{0}, e_{0}\right)$ is called a source (repeller) if $\left|\zeta_{1}\right|>1$ and $\left|\zeta_{2}\right|>1 ;\left(x_{0}, y_{0}, e_{0}\right)$ is called a saddle if $\left|\zeta_{1}\right|>1$ and $\left|\zeta_{2}\right|<1$ (or $\left|\zeta_{1}\right|<1$ and $\left|\zeta_{2}\right|>1$; and $\left(x_{0}, y_{0}, e_{0}\right)$ is called nonhyperbolic if either $\left|\zeta_{1}\right|=1$ or $\left|\zeta_{2}\right|=1$, on the other hand, if $\zeta_{1}=-1$ and $\left|\zeta_{2}\right| \neq 1$ are necessary conditions for the emergence of period-doubling bifurcation and $-2<P<2$ with $Q=1$ are necessary conditions for the occurrence of Neimark-Sacker bifurcation. Moreover, if $S(1)=1+P+Q>0$, then all cases of Lemma 1 are depicted in Figure 2 in $P Q$-plane.

Keeping in view Lemma 1 , the following theorem is presented for local dynamics of system (4) about its positive fixed point.

Theorem 1. Assume that $b>r, b c<r(a p+c)$, and ( $(b d-$ $\left.r(a k+d)) /(b-r)^{2}\right)-(\mu /(r(a p+c)-b c))>0$; then, there exists unique interior (positive) fixed point $\left(x_{0}, y_{0}, e_{0}\right)$ for system (4) satisfying the following conditions:

(i) $\left(x_{0}, y_{0}, e_{0}\right)$ is a sink if and only if $R_{2} \mu<S_{2}$ $((\operatorname{arp} /(b-r))-c)^{2}$ and $R_{3} \mu>\left(S_{3}-2\right)((\operatorname{arp} /(b-$ $r))-c)^{2}$

(ii) $\left(x_{0}, y_{0}, e_{0}\right)$ is a source if and only $R_{2} \mu>S_{2}$ $((\operatorname{arp} /(b-r))-c)^{2}$ and $R_{3} \mu>\left(S_{3}-2\right)((\operatorname{arp} /(b-$ $r))-c)^{2}$

(iii) $\left(x_{0}, y_{0}, e_{0}\right)$ is a saddle if and only if $R_{3} \mu<$ $\left(S_{3}-2\right)((\operatorname{arp} /(b-r))-c)^{2}$

(iv) $\left(x_{0}, y_{0}, e_{0}\right)$ is nonhyperbolic if one of the following conditions hold true:

(1) $R_{3} \mu=\left(S_{3}-2\right)((\operatorname{arp} /(b-r))-c)^{2}, R_{1} \mu \neq\left(S_{1}+\right.$ 2) $((\operatorname{arp} /(b-r))-c)^{2},\left(S_{1}+4\right) \quad((\operatorname{arp} /(b-r))-$ $c)^{2}$

(2) $R_{2} \mu=S_{2}((\operatorname{arp} /(b-r))-c)^{2}$ and $H(\mu)<4$

Next, for $a=3.9, b=14, c=3.7, \quad r=3.4, p=5.8$, $d=21.1$, and $h=0.25$, the dynamical classification of interior equilibrium of system (4) is depicted in $\mu k$-plane (see Figure 3).

Taking into account part (iv.1) of Theorem 1, it is easily to observe that the eigenvalues about the equilibrium point $\left(x_{0}, y_{0}, e_{0}\right)$ are given by $\lambda_{1}=-1$ and $\lambda_{2}=1-P$ with $\left|\lambda_{2}\right| \neq-1$. On the other hand, if part (iv.2) of Theorem 1 holds true, then one can obtain that the eigenvalues for the equilibrium point $\left(x_{0}, y_{0}, e_{0}\right)$ are conjugate complex numbers with modulus one.

Next, we consider the following set:

$$
F_{B}=\left\{\begin{array}{l}
0<\mu=\frac{1}{R_{3}}\left(S_{3}-2\right)\left(\frac{a r p}{b-r}-c\right)^{2}<\left(d-\frac{k a r}{b-r}\right)\left(\frac{p a r}{b-r}-c\right) \\
(a, b, c, d, k, p, r, \mu, h): \\
R_{1} \mu \neq\left(S_{1}+2\right)\left(\frac{a r p}{b-r}-c\right)^{2}, \\
\left(S_{1}+4\right)\left(\frac{\operatorname{arp}}{b-r}-c\right)^{2} .
\end{array}\right.
$$




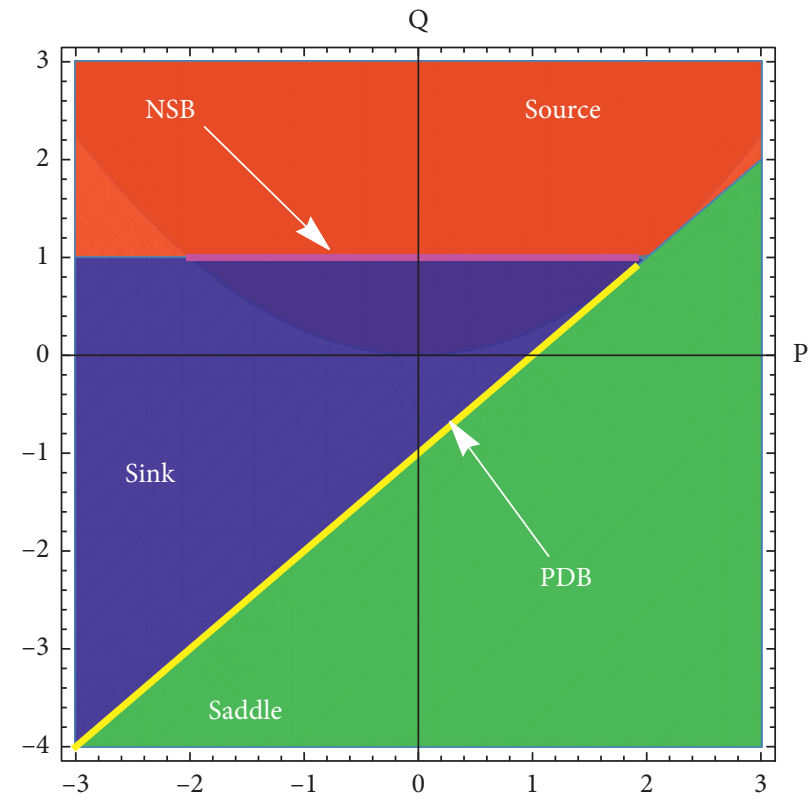

Figure 2: Dynamical classification of planar system with $S(1)>0$.

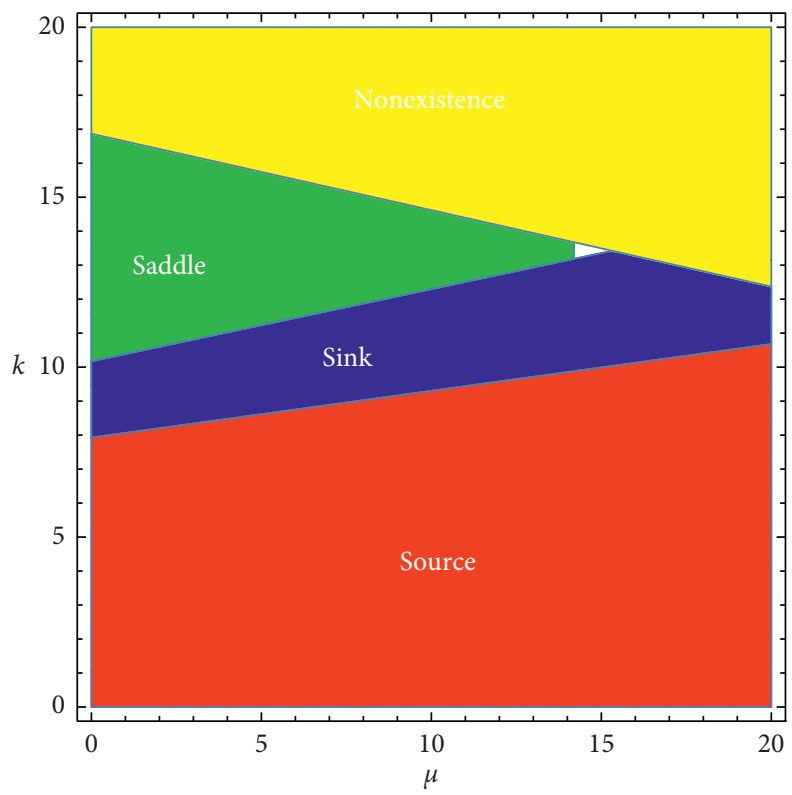

Figure 3: Dynamical classification of interior equilibrium of system (4) in $\mu k$-plane.

It is easy to see that the steady state $\left(x_{0}, y_{0}, e_{0}\right)$ undergoes period-doubling (flip) bifurcation whenever parameters vary in a small neighborhood of $F_{B}$.
Next, we consider the following curve:

$$
\left\{H_{B}=(a, b, c, d, k, p, r, \mu, h): 0<\mu=\frac{S_{2}}{R_{2}}\left(\frac{a r p}{b-r}-c\right)^{2}<\left(d-\frac{k a r}{b-r}\right)\left(\frac{p a r}{b-r}-c\right), H(\mu)<4\right\} .
$$

On the other hand, $\left(x_{0}, y_{0}, e_{0}\right)$ undergoes the Neimark-Sacker (Hopf) bifurcation whenever parameters vary in a small neighborhood of $H_{B}$. In Section 3, we discuss the occurrence of period-doubling bifurcation around the 
interior fixed point $\left(x_{0}, y_{0}, e_{0}\right)$ with the variation of parameters in a small neighborhood of $F_{B}$, and the emergence of the Neimark-Sacker bifurcation about $\left(x_{0}, y_{0}, e_{0}\right)$ with varying the parameters in a small neighborhood of $H_{B}$.

\section{Bifurcation Analysis}

Keeping in view the analysis of Section 3, we discuss the period-doubling bifurcation and Neimark-Sacker bifurcation about the positive fixed point $\left(x_{0}, y_{0}, e_{0}\right)$ in this section. For this, choose parameter $\mu$ as bifurcation parameter for investigating the period-doubling bifurcation and Neimark-Sacker bifurcation about $\left(x_{0}, y_{0}, e_{0}\right)$ by implementing the novel normal form theory of discrete singular systems, the center manifold theorem, and the bifurcation theory of discrete systems [29-35].

3.1. Period-Doubling Bifurcation. First, we start our investigation related to period-doubling bifurcation for system (4) about its equilibrium $\left(x_{0}, y_{0}, e_{0}\right)$ with a variation of parameters in a small neighborhood of $F_{B}$. For this, we choose parameters $\left(a, b, c, d, k, p, r, \mu_{1}, h\right)$ arbitrarily from $F_{B}$, taking into account system (4) with $\left(a, b, c, d, k, p, r, \mu_{1}, h\right) \in F_{B}$. In this case, system (4) is described by the following 2-dimensional map:

$$
\left\{\begin{array}{l}
x \longrightarrow x+h x\left(d-k x-\frac{y}{a+x}-e\right) \\
y \longrightarrow y+h y\left(-r+\frac{b x}{a+x}\right) \\
\mu_{1}=e(p x-c)
\end{array}\right.
$$

It is easy to see that system (17) has a unique positive fixed point $\left(x_{0}, y_{0}, e_{0}\right)$ such that the eigenvalues are given by $\lambda_{1}=-1$ and $\lambda_{2}=1-P$ with $\left|\lambda_{2}\right| \neq-1$. Assume that $\left(a, b, c, d, k, p, r, \mu_{1}, h\right) \in F_{B} \quad$ with $\quad \mu_{1}=\left(1 / R_{1}\right)\left(S_{1}-2\right)$ $((\operatorname{arp} /(b-r))-c)^{2}$. Next, we take $\mu^{*}$ as a bifurcation parameter, considering a perturbation for system (17) as follows:

$$
\left\{\begin{array}{l}
x \longrightarrow x+h x\left(d-k x-\frac{y}{a+x}-e\right) \\
y \longrightarrow y+h y\left(-r+\frac{b x}{a+x}\right) \\
\mu_{1}+\mu^{*}=e(p x-c)
\end{array}\right.
$$

where $\mu^{*} \ll 1$ is taken as a small perturbation parameter.

Then, system (17) can be described in the following way:

$$
\left\{\begin{array}{l}
(x, y)^{T} \longrightarrow f(x, y, e), \\
g(x, y, e)=0
\end{array}\right.
$$

$$
\begin{aligned}
f(x, y, e):= & \left(f_{1}(x, y, e), f_{2}(x, y, e)\right)^{T}, \\
& \left\{\begin{array}{l}
f_{1}(x, y, e)=x+h x\left(d-k x-\frac{y}{a+x}-e\right) \\
f_{2}(x, y, e)=y+h y\left(-r+\frac{b x}{a+x}\right) \\
g(x, y, e)=e(p x-c)-\mu_{1} .
\end{array}\right.
\end{aligned}
$$

Then, it is easy to see that $\operatorname{Dg}\left(x_{0}, y_{0}, e\right)=\left(e_{0} p, 0, p x_{0}-\right.$ c) such that the rank of $\operatorname{Dg}\left(x_{0}, y_{0}, e_{0}\right)=1$. On the other hand, a local parameterization $\psi$ for the 2-dimensional smooth manifold defined by $M_{g}=\left\{(x, y, e) \in R^{3}\right.$; $g(x, y, e)=0$, and $\operatorname{rank} \operatorname{Dg}(x, y, e)=1\}$ about $(x, y, e) \in$ $B\left(x_{0}, y_{0}, e_{0}\right) \subset M_{g}$ is given as follows: for any $(x, y, e) \in B\left(x_{0}, y_{0}, e_{0}\right)$, there is $Z \in A \subset R^{2}$ such that

$$
\begin{aligned}
X & =\psi(Z)=X_{0}+U_{0} Z+V_{0} H(Z), \\
g(\psi(Z)) & =0,
\end{aligned}
$$

where

$$
\begin{aligned}
& U_{0}=\left(\begin{array}{ll}
1 & 0 \\
0 & 1 \\
0 & 0
\end{array}\right), \\
& V_{0}=\left(\begin{array}{l}
0 \\
0 \\
1
\end{array}\right),
\end{aligned}
$$

$X=(x, y, e), \quad X_{0}=\left(x_{0}, y_{0}, e_{0}\right), \quad Z=\left(z_{1}, z_{2}\right)$, and $H: R^{2}$ $\longrightarrow R$ is a smooth mapping. For further details, the interested reader is referred to [36]. Taking into account the definition of $\psi$, we obtain the following:

$$
D \psi(z)=\left(\begin{array}{c}
D(g(X)) \\
U_{0}^{T}
\end{array}\right)^{-1}\left(\begin{array}{ll}
0 & 0 \\
1 & 0 \\
0 & 1
\end{array}\right),
$$

for arbitrarily chosen $(x, y, e) \in B\left(x_{0}, y_{o}, e_{0}\right)$. Then, system (19) is written as follows:

$$
Z \longrightarrow f(Z), \quad Z \in A \subset R^{2},
$$

where $A=\psi^{-1} B\left(x_{0}, y_{o}, e_{0}\right)$.

Taking into account (24), we have

$$
\left\{\begin{array}{l}
z_{1} \longrightarrow a_{1} z_{1}+a_{2} z_{2}+a_{13} z_{1} \mu^{*}+a_{11} z_{1}^{2}+a_{12} z_{1} z_{2}+a_{113} z_{1}^{2} \mu^{*} \\
+a_{111} z_{1}^{3}+a_{112} z_{1}^{2} z_{2}+O\left(\left(\left|z_{1}\right|+\left|z_{2}\right|+\left|\mu^{*}\right|\right)^{4}\right), \\
z_{2} \longrightarrow b_{1} z_{1}+b_{2} z_{2}+b_{13} z_{1} \mu^{*}+b_{11} z_{1}^{2}+b_{12} z_{1} z_{2}+b_{113} z_{1}^{2} \mu^{*} \\
+b_{111} z_{1}^{3}+b_{112} z_{1}^{2} z_{2}+O\left(\left(\left|z_{1}\right|+\left|z_{2}\right|+\left|\mu^{*}\right|\right)^{4}\right) .
\end{array}\right.
$$

where 
In order to compute the coefficients related the normal form, we need some further computation. From the aforementioned computation, one can easily get

$$
\begin{aligned}
D f_{1}(X) & =\left(1+h\left(d-2 k x-\frac{a y}{(a+x)^{2}}-e\right),-\frac{h x}{a+x},-h x\right) \\
D f_{2}(X) & =\left(\frac{a b h y}{(a+x)^{2}}, 1+h\left(-r+\frac{b x}{a+x}\right), 0\right) \\
D g(X) & =(e p, 0, p x-c) \\
D \psi(z) & =\left(\begin{array}{cc}
D g(X) \\
U_{0}^{T}
\end{array}\right)-1\left(\begin{array}{cc}
0 & 0 \\
1 & 0 \\
0 & 1
\end{array}\right)=\left(\begin{array}{ccc}
e p & 0 & p x-c \\
1 & 0 & 0 \\
0 & 1 & 0
\end{array}\right)^{-1}\left(\begin{array}{ll}
0 & 0 \\
1 & 0 \\
1 & 0 \\
0 & 1
\end{array}\right) \\
& =\left(\begin{array}{cc}
0 & 1 \\
\frac{e p}{p x-c} & 0
\end{array}\right)=\left(\begin{array}{cc}
\left.D_{z_{1}} \psi(z), D_{z_{2}} \psi(z)\right),
\end{array}\right)
\end{aligned}
$$

and from this, it follows that

$$
\begin{aligned}
& D_{z_{1}} \psi(z)=\left(1,0,-\frac{e p}{p x-c}\right)^{T}, \\
& D_{z_{2}} \psi(z)=(0,1,0)^{T} .
\end{aligned}
$$

Assume that $f_{i z_{i}}(\psi(Z))$ represents the derivative of $f_{i}(\psi(Z))$ with respect to $z_{i}$ and taking $f_{i z_{i}}(X)=f_{i z_{i}}(\psi(Z))$. In a similar way, one can adopt notations for $f_{i z_{i} z_{j}}(\psi(Z))$ and $f_{i z_{i} z_{j} z_{k}}(\psi(Z))$. Next, it is easy to see that

$$
\left\{\begin{array}{l}
f_{1 z_{1}}(X)=D f_{1}(X) D_{z_{1}} \psi(z)=1+h\left(d-2 k x-\frac{a y}{(a+x)^{2}}-e+\frac{e p x}{p x-c}\right) \\
f_{1 z_{2}}(X)=D f_{1}(X) D_{z_{2}} \psi(z)=-\frac{h x}{a+x} \\
f_{2 z_{1}}(X)=D f_{2}(X) D_{z_{1}} \psi(z)=\frac{a b h y}{(a+x)^{2}}, \\
f_{2 z_{2}}(X)=D f_{2}(X) D_{z_{2}} \psi(z)=1+h\left(-r+\frac{b x}{a+x}\right)
\end{array}\right.
$$

Putting $X_{0}$ into (28), one has the following: 


$$
\left\{\begin{array}{l}
f_{1 z_{1}}\left(X_{0}\right)=1+h\left(-k x_{0}+\frac{e_{0} p x_{0}}{p x_{0}-c}+\frac{x_{0}\left(d-k x_{0}-e_{0}\right)}{a+x_{0}}\right)=1+\frac{h r}{b}\left(d+\frac{a k(b+r)}{b-r}\right) \\
+\frac{h r}{b} \frac{(b-r)^{2}(a p+c)}{(a p r-c b+c r)^{2}}\left(\mu_{1}+\mu^{*}\right), \\
f_{1 z_{2}}\left(X_{0}\right)=-\frac{x_{0}}{a+x_{0}}=-\frac{h r}{b}, \\
f_{2 z_{1}}\left(X_{0}\right)=\frac{a b h y_{0}}{\left(a+x_{0}\right)^{2}}=\frac{a b h\left(d-k x_{0}-e_{0}\right)}{a+x_{0}}=h(b-r)\left(d-\frac{k a r}{b-r}-\frac{\left(\mu_{1}+\mu_{*}\right)(b-r)}{a p r-c b+c r}\right) \\
f_{2 z_{2}}\left(X_{0}\right)=1+h\left(-r+\frac{b x_{0}}{a+x_{0}}\right)=1 .
\end{array}\right.
$$

Then, from (28), it is easy to see that

$$
\left\{\begin{array}{l}
D f_{1 z_{1}}(X)=\left(h\left(-2 k+\frac{2 a y}{(a+x)^{3}}-\frac{e p c}{(p x-c)^{2}}\right),-\frac{a h}{(a+x)^{2}}, h\left(-1+\frac{p x}{p x-c}\right)\right) \\
D f_{1 z_{2}}(X)=\left(-\frac{a h}{(a+x)^{2}}, 0,0\right) \\
D f_{2 z_{1}}(X)=\left(-\frac{2 a b h y}{(a+x)^{3}}, \frac{a b h}{(a+x)^{2}}, 0\right) \\
D f_{2 z_{2}}(X)=\left(\frac{a b h}{(a+x)^{2}}, 0,0\right) .
\end{array}\right.
$$

Consequently, one has the following:

$$
\left\{\begin{array}{l}
f_{1 z_{1} z_{1}}(X)=D f_{1 z_{1}}(X) D_{z_{1}} \psi(z)=h\left[-2 k+\frac{2 a y}{(a+x)^{3}}-\frac{2 e p c}{(p x-c)^{2}}\right] \\
f_{1 z_{1} z_{2}}(X)=D f_{1 z_{1}}(X) D_{z_{2}} \psi(z)=-\frac{a h}{(a+x)^{2}}, \\
f_{2 z_{1} z_{1}}(X)=D f_{2 z_{1}}(X) D_{z_{1}} \psi(z)=-\frac{2 a b h y}{(a+x)^{3}} \\
f_{2 z_{1} z_{2}}(X)=D f_{2 z_{1}}(X) D_{z_{2}} \psi(z)=\frac{a b h}{(a+x)^{2}} .
\end{array}\right.
$$


Putting the value of $X_{0}$ in (31), one has the following:

$$
\left\{\begin{array}{l}
f_{1 z_{1} z_{1}}\left(X_{0}\right)=h\left[-2 k+\frac{2 a\left(d-k x_{0}-e_{0}\right)}{\left(a+x_{0}\right)^{2}}-\frac{2 e_{0} p c}{\left(p x_{0}-c\right)^{2}}\right]=h\left[-2 k+\frac{2 d(b-r)^{2}}{a b^{2}}-\frac{2 k r(b-r)}{b^{2}}\right] \\
\left.\frac{2(b-r)^{3}}{a p r-c b+c r}\left(\frac{1}{a b^{2}}+\frac{p c}{(a p r-c b+c r)^{2}}\right)\left(\mu_{1}+\mu^{*}\right)\right], \\
f_{1 z_{1} z_{2}}\left(X_{0}\right)=-\frac{a h}{\left(a+x_{0}\right)^{2}}=-\frac{h(b-r)^{2}}{a b^{2}}, \\
f_{2 z_{1} z_{1}}\left(X_{0}\right)=-\frac{2 a b h\left(d-k x_{0}-e_{0}\right)}{\left(a+x_{0}\right)^{2}}=\frac{2 h(b-r)^{2}}{a b}\left(\frac{a k r}{b-r}-d\right)+\frac{2 h(b-r)^{3}}{a b(a p r-c b+c r)}\left(\mu_{1}+\mu^{*}\right), \\
f_{2 z_{1} z_{2}}\left(X_{0}\right)=\frac{a b h}{\left(a+x_{0}\right)^{2}}=\frac{h(b-r)^{2}}{a b} .
\end{array}\right.
$$

Then, from (31), it follows that

$$
\left\{\begin{array}{l}
D f_{1 z_{1} z_{1}}(X)=\left(-\frac{6 a h y}{(a+x)^{4}}+\frac{4 h e p^{2} c}{(p x-c)^{3}}, \frac{2 a h}{(a+x)^{3}},-\frac{2 h p c}{(p x-c)^{2}}\right), \\
D f_{1 z_{1} z_{2}}(X)=\left(\frac{2 a h}{(a+x)^{3}}, 0,0\right), \\
D f_{2 z_{1} z_{1}}(X)=\left(\frac{6 a b h y}{(a+x)^{4}},-\frac{2 a b h}{(a+x)^{3}}, 0\right), \\
D f_{2 z_{1} z_{2}}(X)=\left(-\frac{2 a b h}{(a+x)^{3}}, 0,0\right) .
\end{array}\right.
$$


Then, we get

$$
\left\{\begin{array}{l}
f_{1 z_{1} z_{1} z_{1}}(X)=D f_{1 z_{1} z_{1}}(X) D_{z_{1}} \psi(z)=-\frac{6 a h y}{(a+x)^{4}}+\frac{6 h e p^{2} c}{(p x-c)^{3}} \\
f_{1 z_{1} z_{1} z_{2}}(X)=D f_{1 z_{1} z_{1}}(X) D_{z_{2}} \psi(z)=\frac{2 a h}{(a+x)^{3}}, \\
f_{2 z_{1} z_{1} z_{1}}(X)=D f_{2 z_{1} z_{1}}(X) D_{z_{1}} \psi(z)=\frac{6 a b h y}{(a+x)^{4}} \\
f_{2 z_{1} z_{1} z_{2}}(X)=D f_{2 z_{1} z_{1}}(X) D_{z_{2}} \psi(z)=-\frac{2 a b h}{(a+x)^{3}} .
\end{array}\right.
$$

Putting the value of $X_{0}$ into (34), we obtain that

$$
\left\{\begin{array}{l}
f_{1 z_{1} z_{1} z_{1}}\left(X_{0}\right)=-\frac{6 a h\left(d-k x_{0}-e_{0}\right)}{\left(a+x_{0}\right)^{3}}+\frac{6 h e_{0} p^{2} c}{\left(p x_{0}-c\right)^{3}}=\frac{6 h k r(b-r)^{2}}{a b^{3}}-\frac{6 h d(b-r)^{3}}{a^{2} b^{3}} \\
+\frac{6 h(b-r)^{4}}{a p r-c b+c r}\left[\frac{1}{a^{2} b^{3}}+\frac{p^{2} c}{(a p r-c b+c r)^{3}}\right]\left(\mu_{1}+\mu^{*}\right) \\
f_{1 z_{1} z_{1} z_{2}}\left(X_{0}\right)=\frac{2 a h}{\left(a+x_{0}\right)^{3}}=\frac{2 h(b-r)^{3}}{a^{2} b^{3}}, \\
f_{2 z_{1} z_{1} z_{1}}\left(X_{0}\right)=\frac{6 a b h\left(d-k x_{0}-e_{0}\right)}{\left(a+x_{0}\right)^{3}}=\frac{6 h(b-r)^{3}}{a^{2} b^{2}}\left(d-\frac{a k r}{b-r}\right)-\frac{6 h(b-r)^{4}}{a^{2} b^{2}(a p r-c b+c r)}\left(\mu_{1}+\mu^{*}\right) \\
f_{2 z_{1} z_{1} z_{2}}\left(X_{0}\right)=-\frac{2 a b h}{\left(a+x_{0}\right)^{3}}=-\frac{2 h(b-r)^{3}}{a^{2} b^{2}} .
\end{array}\right.
$$


Consequently, it follows from (29), (32), and (35) that

$$
\begin{aligned}
& a_{1}=1+\frac{h r}{b}\left(d+\frac{a k(b+r)}{b-r}\right)+\frac{h r}{b} \frac{(b-r)^{2}(a p+c)}{(a p r-c b+c r)^{2}} \mu_{1} \\
& a_{2}=-\frac{h r}{b}, \\
& a_{13}=\frac{h r}{b} \frac{(b-r)^{2}(a p+c)}{(a p r-c b+c r)^{2}} \\
& a_{11}=h\left[-2 k+\frac{2 d(b-r)^{2}}{a b^{2}}-\frac{2 k r(b-r)}{b^{2}}-\frac{2(b-r)^{3}}{a p r-c b+c r}\left(\frac{1}{a b^{2}}+\frac{p c}{(a p r-c b+c r)^{2}}\right) \mu_{1}\right], \\
& a_{12}=-\frac{h(b-r)^{2}}{a b^{2}} \\
& a_{113}=-\frac{2 h(b-r)^{3}}{a p r-c b+c r}\left[\frac{1}{a b^{2}}+\frac{p c}{(a p r-c b+c r)^{2}}\right] \\
& a_{111}=\frac{6 h k r(b-r)^{2}}{a b^{3}}-\frac{6 h d(b-r)^{3}}{a^{2} b^{3}}+\frac{6 h(b-r)^{4}}{a p r-c b+c r}\left[\frac{1}{a^{2} b^{3}}+\frac{p^{2} c}{(a p r-c b+c r)^{3}}\right] \mu_{1} \text {, } \\
& a_{112}=\frac{2 h(b-r)^{3}}{a^{2} b^{3}} \\
& b_{1}=h(b-r)\left(d-\frac{b-r}{a p r-c b+c r} \mu_{1}\right)-a k r \\
& b_{2}=1 \text {, } \\
& b_{13}=-\frac{h(b-r)^{2}}{a p r-c b+c r} \\
& b_{11}=\frac{2 h(b-r)^{2}}{a b}\left(\frac{a k r}{b-r}-d\right)+\frac{2 h(b-r)^{3}}{a b(a p r-c b+c r)} \mu_{1} \text {, } \\
& b_{12}=\frac{h(b-r)^{2}}{a b} \\
& b_{113}=\frac{2 h(b-r)^{3}}{a b(a p r-c b+c r)} \\
& b_{111}=\frac{6 h(b-r)^{3}}{a^{2} b^{2}}\left(d-\frac{a k r}{b-r}\right)-\frac{6 h(b-r)^{4}}{a^{2} b^{2}(a p r-c b+c r)} \mu_{1}, \\
& b_{112}=-\frac{2 h(b-r)^{3}}{a^{2} b^{2}}
\end{aligned}
$$


Next, we consider the following nonsingular matrix:

$$
T=\left(\begin{array}{cc}
a_{2} & a_{2} \\
-1-a_{1} & \lambda_{2}-a_{1}
\end{array}\right),
$$

implementing the following translation:

$$
\left(\begin{array}{c}
z_{1} \\
z_{2}
\end{array}\right)=T\left(\begin{array}{l}
u \\
v
\end{array}\right)
$$

Then, it is easy to see that system (24) can be written as follows:

$$
\left(\begin{array}{l}
u \\
v
\end{array}\right) \longrightarrow\left(\begin{array}{cc}
-1 & 0 \\
0 & \lambda_{2}
\end{array}\right)\left(\begin{array}{l}
u \\
v
\end{array}\right)+\left(\begin{array}{l}
f_{1}\left(u, v, \mu^{*}\right) \\
f_{2}\left(u, v, \mu^{*}\right)
\end{array}\right)
$$

where

$$
\begin{aligned}
& f_{1}\left(u, v, \mu^{*}\right)=\frac{a_{11}\left(\lambda_{2}-a_{1}\right)-a_{2} b_{11}}{a_{2}\left(1+\lambda_{2}\right)} z_{1}^{2}+\frac{a_{12}\left(\lambda_{2}-a_{1}\right)-a_{2} b_{12}}{a_{2}\left(1+\lambda_{2}\right)} z_{1} z_{2}+\frac{a_{13}\left(\lambda_{2}-a_{1}\right)-a_{2} b_{13}}{a_{2}\left(1+\lambda_{2}\right)} z_{1} \mu^{*} \\
& +\frac{a_{113}\left(\lambda_{2}-a_{1}\right)-a_{2} b_{113}}{a_{2}\left(1+\lambda_{2}\right)} z_{1}^{2} \mu^{*}+\frac{a_{111}\left(\lambda_{2}-a_{1}\right)-a_{2} b_{111}}{a_{2}\left(1+\lambda_{2}\right)} z_{1}^{3} \\
& +\frac{a_{112}\left(\lambda_{2}-a_{1}\right)-a_{2} b_{112}}{a_{2}\left(1+\lambda_{2}\right)} z_{1}^{2} z_{2}+O\left(\left(\left|z_{1}\right|+\left|z_{2}\right|+\left|\mu^{*}\right|\right)^{4}\right) \\
& f_{2}\left(u, v, \mu^{*}\right)=\frac{a_{11}\left(1+a_{1}\right)+a_{2} b_{11}}{a_{2}\left(1+\lambda_{2}\right)} z_{1}^{2}+\frac{a_{12}\left(1+a_{1}\right)+a_{2} b_{12}}{a_{2}\left(1+\lambda_{2}\right)} z_{1} z_{2}+\frac{a_{13}\left(1+a_{1}\right)+a_{2} b_{13}}{a_{2}\left(1+\lambda_{2}\right)} z_{1} \mu^{*} \\
& +\frac{a_{113}\left(1+a_{1}\right)+a_{2} b_{113}}{a_{2}\left(1+\lambda_{2}\right)} z_{1}^{2} \mu^{*}+\frac{a_{111}\left(1+a_{1}\right)+a_{2} b_{111}}{a_{2}\left(1+\lambda_{2}\right)} z_{1}^{3} \\
& +\frac{a_{112}\left(1+a_{1}\right)+a_{2} b_{112}}{a_{2}\left(1+\lambda_{2}\right)} z_{1}^{2} z_{2}+O\left(\left(\left|z_{1}\right|+\left|z_{2}\right|+\left|\mu^{*}\right|\right)^{4}\right) \\
& z_{1}=a_{2}(u+v), z_{2}=-\left(1+a_{1}\right) u+\left(\lambda_{2}-a_{1}\right) v \\
& z_{1} z_{2}=a_{2}\left[-\left(1+a_{1}\right) u^{2}+\left(\lambda_{2}-2 a_{1}-1\right) u v+a_{2}\left(\lambda_{2}-a_{1}\right) v^{2}\right] \\
& z_{1}^{2}=a_{2}^{2}\left[u^{2}+u v+v^{2}\right] \\
& z_{1}^{3}=a_{2}^{3}\left[u^{3}+3 u^{2} v+3 u v^{2}+v^{3}\right] \\
& z_{1}^{2} z_{2}=a_{2}^{2}\left[-\left(1+a_{1}\right) u^{3}+\left(\lambda_{2}-3 a_{1}-2\right) u^{2} v+\left(2 \lambda_{2}-3 a_{1}-1\right) u v^{2}+\left(\lambda_{2}-1\right) v^{3}\right] \text {. }
\end{aligned}
$$

In order to determine the center manifold $W_{c}(0,0,0)$ of (39) about the equilibrium point $(0,0)$ in a small neighborhood of $\mu^{*}$, we implement an application of the center manifold theorem [29], and it is easy to see that there exists a center manifold of the following form:

$$
W_{c}(0,0,0)=\left\{\left(u, v, \mu^{*}\right) \in R^{3}, v=h\left(u, \mu^{*}\right), h(0,0)=0, D h(0,0)=0\right\},
$$
that

for $u$ and $\mu^{*}$ sufficiently small. Furthermore, we assume

$$
h\left(u, \mu^{*}\right)=c_{0} u^{2}+c_{1} u \mu^{*}+c_{2} \mu^{* 2}+O\left(\left(|u|+\left|\mu^{*}\right|\right)^{3}\right) .
$$

Then, it is easy to see that the center manifold satisfies the following:

$$
h\left(-u+f_{1}\left(u, h\left(u, \mu^{*}\right), \mu^{*}\right), \mu^{*}\right)=\lambda_{2} h\left(u, \mu^{*}\right)+f_{2}\left(u, h\left(u, \mu^{*}\right), \mu^{*}\right) .
$$


Taking into account (42) and (43) and then comparing coefficients for (43), one can easily obtain that

$$
\begin{aligned}
& c_{0}=\frac{1}{\left(1-\lambda_{2}^{2}\right)}\left[\left(1+a_{1}\right)\left(a_{2} a_{11}-a_{12}\left(1+a_{1}\right)-a_{2} b_{12}\right)+a_{2}^{2} b_{11}\right] \\
& c_{1}=\frac{a_{13}\left(1+a_{1}\right)+a_{2} b_{13}}{\left(1+\lambda_{2}\right)^{2}} \\
& c_{2}=0 .
\end{aligned}
$$

Furthermore, taking into account (39), it is restricted to the center manifold $W_{c}(0,0,0)$ as follows:

$$
F: u \longrightarrow-u+d_{11} u^{2}+d_{12} u \mu^{*}+d_{112} u^{2} \mu^{*}+d_{122} u \mu^{* 2}+d_{111} u^{3}+O\left(\left(|u|+\left|\mu^{*}\right|\right)^{4}\right)
$$

where

$$
\begin{aligned}
d_{11}= & \frac{1}{\lambda_{2}+1}\left[\left(\lambda_{2}-a_{1}\right)\left(a_{11} a_{2}-a_{12}\left(1+a_{1}\right)+a_{113} a_{2}\right)-b_{11} a_{2}^{2}+\left(1+a_{1}\right) b_{12} a_{2}-b_{113} a_{2}^{2}\right], \\
d_{12}= & \frac{1}{\lambda_{2}+1}\left[a_{13}\left(\lambda_{2}-1\right)-b_{13} a_{2}\right], \\
d_{112}= & \frac{1}{\lambda_{2}+1}\left[\left(\lambda_{2}-a_{1}\right)\left(a_{11} a_{2} c_{1}+a_{12} c_{1}\left(\lambda_{2}-2 a_{1}-1\right)+a_{13} c_{0}\right)-b_{11} a_{2}^{2} c_{1}\right] \\
& \left.-b_{12} a_{2} c_{1}\left(\lambda_{2}-2 a_{1}-1\right)-b_{13} a_{2} c_{0}\right], \\
d_{122}= & \frac{1}{\lambda_{2}+1}\left[a_{13} c_{1}\left(\lambda_{2}-1\right)-b_{13} a_{2} c_{1}\right], \\
d_{111}= & \frac{1}{\lambda_{2}+1}\left[\left(\lambda_{2}-a_{1}\right)\left(a_{11} a_{2} c_{0}+a_{111} a_{2}^{2}+a_{12} c_{0}\left(\lambda_{2}-2 a_{1}-1\right)-a_{112} a_{2}\left(1+a_{1}\right)\right)-b_{11} a_{2}^{2} c_{0}\right] \\
& \left.-b_{12} a_{2} c_{0}\left(\lambda_{2}-2 a_{1}-1\right)-b_{111} a_{2}^{3}+b_{112} a_{2}^{2}\left(1+a_{1}\right)\right] .
\end{aligned}
$$

In order for system (45) to undergo a flip bifurcation, we require that two discriminatory quantities $\alpha_{1}$ and $\alpha_{2}$ are not zero, where

$$
\begin{aligned}
& \alpha_{1}=\left(2 \frac{\partial^{2} F}{\partial \mu^{*} \partial u}+\frac{\partial F}{\partial \mu^{*}} \frac{\partial F}{\partial u}\right)_{(0,0)}=2 d_{12}, \\
& \alpha_{2}=\left(\frac{1}{2}\left(\frac{\partial^{2} F}{\partial u^{2}}\right)^{2}+\frac{1}{3}\left(\frac{\partial^{3} F}{\partial u^{3}}\right)\right)_{(0,0)}=2\left(d_{111}+d_{11}^{2}\right) .
\end{aligned}
$$

Keeping in view the aforementioned computation and bifurcation theory given in $[36,37]$, we have the following theorem.

Theorem 2. Assume that $\left(a, b, c, d, k, p, r, \mu_{1}\right) \in F_{B}$. If $\alpha_{2} \neq 0$, then system (17) undergoes a flip bifurcation at the fixed point $\left(x_{0}, y_{0}, e_{0}\right)$ when the parameter $\mu$ varies in a small neighborhood of $\mu_{1}$. Moreover, if $\alpha_{2}>0$ (resp., $\left.\alpha_{2}<0\right)$, then the period-2 orbits that bifurcate from $\left(x_{0}, y_{0}, e_{0}\right)$ are stable (resp., unstable). 
In the last Section 5 related to numerical simulation, we choose some parametric values for system (4) such that it undergoes period-doubling bifurcation about positive equilibrium as $\mu$ varies in the suitable interval (see Figure 4).

3.2. Neimark-Sacker Bifurcation. Finally, we discuss the Neimark-Sacker bifurcation about equilibrium $\left(x_{0}, y_{0}, e_{0}\right)$ with variation of parameters $\left(a, b, c, d, k, p, r, \mu_{2}, h\right)$ in a small neighborhood of $H_{B}$. For this, we choose parameters $\left(a, b, c, d, k, p, r, \mu_{2}, h\right)$ arbitrarily from $H_{B}$, taking into account system (4) with $\left(a, b, c, d, k, p, r, \mu_{2}, h\right) \in H_{B}$. In this case, system (4) is described by the following 2 -dimensional map:

$$
\left\{\begin{array}{l}
x \longrightarrow x+x\left(d-k x-\frac{y}{a+x}-e\right), \\
y \longrightarrow y+y\left(-r+\frac{b x}{a+x}\right), \\
\mu_{2}=e(p x-c) .
\end{array}\right.
$$

Then, it is easy to observe that map (48) has a unique positive fixed point $\left(x_{0}, y_{0}, e_{0}\right)$. Next, we take $\mu^{*}$ as a bifurcation parameter and consider a perturbation corresponding to map (48) given as follows:

$$
\left\{\begin{array}{l}
x \longrightarrow x+x\left(d-k x-\frac{y}{a+x}-e\right), \\
y \longrightarrow y+y\left(-r+\frac{b x}{a+x}\right) \\
\mu_{2}+\mu^{*}=e(p x-c)
\end{array}\right.
$$

where $\mu^{*} \ll 1$ is taken as a small perturbation parameter.

Furthermore, from (24), we have

$$
\left\{\begin{array}{l}
z_{1} \longrightarrow a_{1} z_{1}+a_{2} z_{2}+a_{11} z_{1}^{2}+a_{12} z_{1} z_{2}+a_{111} z_{1}^{3}+a_{112} z_{1}^{2} z_{2}+O\left(\left(\left|z_{1}\right|+\left|z_{2}\right|\right)^{4}\right) \\
z_{2} \longrightarrow b_{1} z_{1}+b_{2} z_{2}+b_{11} z_{1}^{2}+b_{12} z_{1} z_{2}+b_{111} z_{1}^{3}+b_{112} z_{1}^{2} z_{2}+O\left(\left(\left|z_{1}\right|+\left|z_{2}\right|\right)^{4}\right)
\end{array}\right.
$$

where coefficients $a_{1}, a_{2}, a_{11}, a_{12}, a_{111}, a_{112}$ and $b_{1}, b_{2}, b_{11}, b_{12}, b_{111}, b_{112}$ given in (24) are similar to the coefficients in (36) by replacing $\mu_{1}$ with $\mu_{2}+\mu^{*}$. Moreover, it must be noted that the characteristic equation corresponding to the linearization of $(50)$ about $\left(z_{1}, z_{2}\right)=(0,0)$ is given as follows:

$$
\lambda^{2}+p\left(\mu^{*}\right) \lambda+q\left(\mu^{*}\right)=0
$$

where

$$
\begin{aligned}
& p\left(\mu^{*}\right)=h\left(\mu^{*}\right)-q\left(\mu^{*}\right)-1, \\
& q\left(\mu^{*}\right)=\frac{1}{((\operatorname{arp} /(b-r))-c)^{2}}\left[R_{2} \mu-\left(S_{2}-1\right)\left(\frac{\operatorname{arp}}{b-r}-c\right)^{2}\right], \\
& h\left(\mu^{*}\right)=\frac{a b x_{0} y_{0}}{\left(a+x_{0}\right)^{3}}>0 .
\end{aligned}
$$

Suppose that $\left(a, b, c, d, k, p, r, \mu_{2}, h\right) \in H_{B}$; then eigenvalues about $(0,0)$ are conjugate complex numbers denoted by $\lambda$ and $\bar{\lambda}$ with modulus 1 such that

$$
\lambda, \bar{\lambda}=-\frac{p\left(\mu^{*}\right)}{2} \pm \frac{i}{2} \sqrt{4 q\left(\mu^{*}\right)-p^{2}\left(\mu^{*}\right)},
$$

and, consequently, one has

$$
\begin{gathered}
|\lambda|_{\mu^{*}=0}=\sqrt{q(0)}=1, \\
l=\left.\frac{d|\lambda|}{d \mu^{*}}\right|_{\mu^{*}=0}=\frac{R_{2}}{2\left(r_{1}-c\right)^{2}} \neq 0 .
\end{gathered}
$$

On the other hand, it is necessary that, at $\mu^{*}=0$, one must have $\lambda^{m}, \bar{\lambda}^{m} \neq 1,(m=1,2,3,4)$, or equivalently one has $p(0) \neq-2,0,1,2$. Next, it must be noted that $p(0)=h(0)-$ $q(0)-1=H\left(\mu_{2}\right)-2$ and $H\left(\mu_{2}\right)<4$ because $(a, b, c, d, k$, $\left.p, r, \mu_{2}, h\right) \in H_{B}$. As a result, one has $p(0) \neq-2,2$. Consequently, it is required that $p(0) \neq 0,1$, which is equivalently written as follows:

$$
H\left(\mu_{2}\right) \neq 2,3
$$

Consequently, the eigenvalues of system (50) which are given by $\lambda, \bar{\lambda}$ about fixed point $(0,0)$ do not lie in the intersection of the unit circle with the coordinate axes at $\mu^{*}=0$ whenever conditions (55) hold true. Moreover, in order to discuss the normal form for system (50) about $\mu^{*}=0$, we choose $\quad \mu^{*}=0, \quad \sigma=1-\left(H\left(\mu_{2}\right) / 2\right), \quad \omega=(1 / 2)$ $\sqrt{H\left(\mu_{2}\right)\left(4-H\left(\mu_{2}\right)\right)}$,

$$
T=\left(\begin{array}{cc}
a_{2} & 0 \\
\sigma-a_{1} & -\omega
\end{array}\right),
$$

taking into account the following translation: 


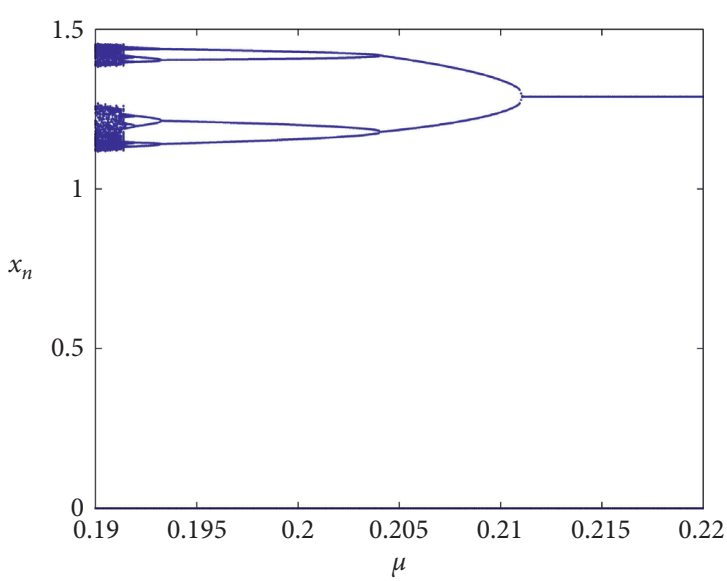

(a)

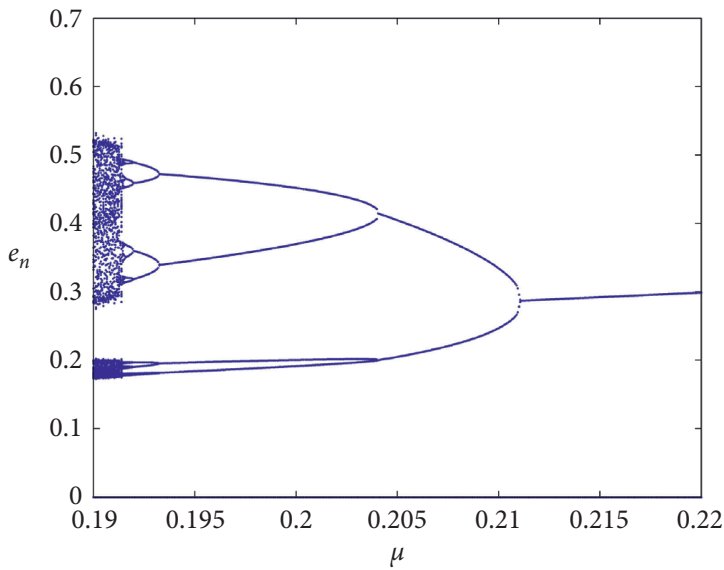

(c)

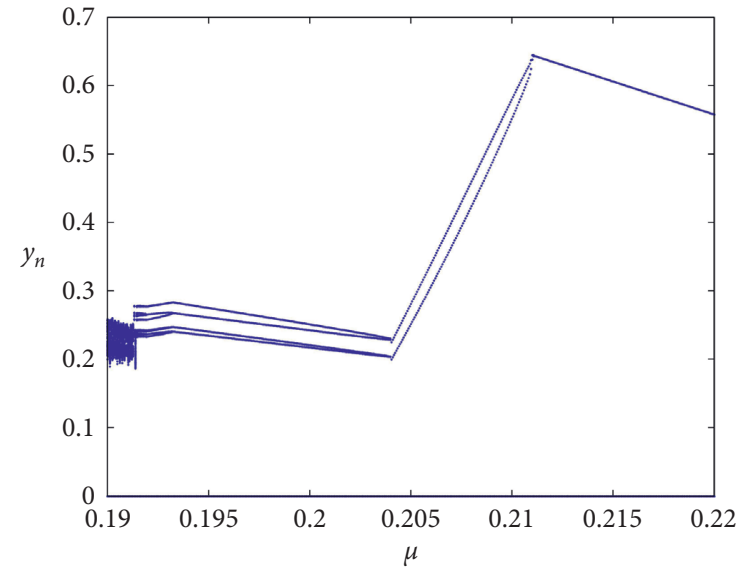

(b)

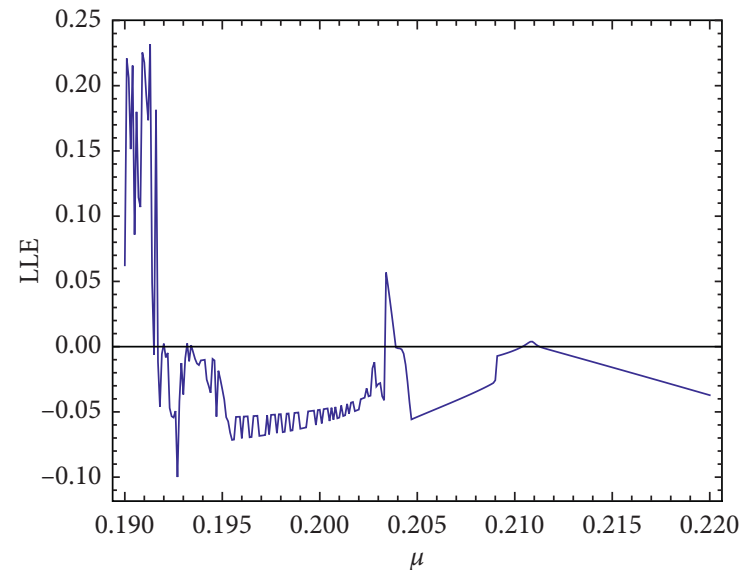

(d)

Figure 4: Bifurcation diagrams and LLE for system (3) with $a=5.8, b=8.8, c=2.1, d=3.6, k=2.5, r=1.6, p=2.2, h=0.98$, $\mu \in[0.19,0.22]$, and $\left(x_{0}, y_{0}, e_{0}\right)=(1.28,0.6428,0.287)$. (a) Bifurcation diagram for $x_{n}$. (b) Bifurcation diagram for $y_{n}$. (c) Bifurcation diagram for $e_{n}$. (d) Largest Lyapunov exponents.

$$
\left(\begin{array}{c}
z_{1} \\
z_{2}
\end{array}\right)=T\left(\begin{array}{l}
u \\
v
\end{array}\right)
$$

$$
\left(\begin{array}{l}
u \\
v
\end{array}\right) \longrightarrow\left(\begin{array}{cc}
\sigma & -\omega \\
\omega & \sigma
\end{array}\right)\left(\begin{array}{l}
u \\
v
\end{array}\right)+\left(\begin{array}{l}
\overline{f_{1}}(u, v) \\
\overline{f_{2}}(u, v)
\end{array}\right)
$$

Then, it is easy to see that system (50) takes the following form:

where

$$
\begin{aligned}
\overline{f_{1}}(u, v)= & \frac{a_{11}}{a_{2}} z_{1}^{2}+\frac{a_{12}}{a_{2}} z_{1} z_{2}+\frac{a_{111}}{a_{2}} z_{1}^{3}+\frac{a_{112}}{a_{2}} z_{1}^{2} z_{2}+O\left(\left(\left|z_{1}\right|+\left|z_{2}\right|\right)^{4}\right) \\
\overline{f_{2}}(u, v)= & \frac{\left(\sigma-a_{1}\right) a_{11}-a_{2} b_{11}}{a_{2} \omega} z_{1}^{2}+\frac{\left(\sigma-a_{1}\right) a_{12}-a_{2} b_{12}}{a_{2} \omega} z_{1} z_{2} \\
& +\frac{\left(\sigma-a_{1}\right) a_{111}-a_{2} b_{111}}{a_{2} \omega} z_{1}^{3}+\frac{\left(\sigma-a_{1}\right) a_{112}-a_{2} b_{112}}{a_{2} \omega} z_{1}^{2} z_{2}+O\left(\left(\left|z_{1}\right|+\left|z_{2}\right|\right)^{4}\right) \\
z_{1}= & a_{2} u, \\
z_{2}= & \left(\sigma-a_{1}\right) u-\omega v,
\end{aligned}
$$




$$
\begin{aligned}
z_{1} z_{2} & =a_{2}\left(\sigma-a_{1}\right) u^{2}+a_{2} \omega u v \\
z_{1}^{2} & =a_{2}^{2} u^{2} \\
z_{1}^{3} & =a_{2}^{3} u^{3} \\
z_{1}^{2} z_{2} & =a_{2}^{2}\left(\sigma-a_{1}\right) u^{3}-\omega u^{2} v .
\end{aligned}
$$

Therefore, at point $(0,0)$, we have

$$
\begin{aligned}
& \bar{f}_{1 u u}=2 a_{11}+2 a_{12}\left(\sigma-a_{1}\right), \\
& \bar{f}_{1 u v}=a_{12} \omega, \\
& \bar{f}_{1 u u u}=6 a_{111} a_{2}^{2}, \\
& \bar{f}_{1 u u v}=-\frac{a_{112}}{a_{2}} \omega, \\
& \bar{f}_{1 v v}=\bar{f}_{1 u v v}=\bar{f}_{1 v v v}=0, \\
& \bar{f}_{2 u u}=\frac{2}{\omega}\left[\left(\sigma-a_{1}\right)\left(a_{11} a_{2}+a_{12}\left(\sigma-a_{1}\right)-a_{2} b_{12}\right)-a_{2}^{2} b_{11}\right], \\
& \bar{f}_{2 u v}=\left(\sigma-a_{1}\right) a_{12}-a_{2} b_{12}, \\
& \bar{f}_{2 u u u}=\frac{6 a_{2}}{\omega}\left[\left(\sigma-a_{1}\right)\left(a_{2} a_{111}+\left(\sigma-a_{1}\right) a_{112}-a_{2} b_{112}\right)-a_{2} b_{111}\right], \\
& \bar{f}_{2 u u v}=\frac{2}{a_{2}}\left[a_{2} b_{112}-\left(\sigma-a_{1}\right) a_{112}\right], \\
& \bar{f}_{2 v v}=\bar{f}_{2 u v v}=\bar{f}_{2 v v v}=0 .
\end{aligned}
$$

Then, system (58) undergoes a Neimark-Sacker bifurcation, if we have the following nonzero discriminatory quantity [30,32]:

$$
\theta=\left[-\operatorname{Re}\left(\frac{(1-2 \lambda) \bar{\lambda}^{2}}{1-\lambda}\right) L_{11} L_{12}-\frac{1}{2}\left|L_{11}\right|^{2}-\left|L_{21}\right|^{2}+\operatorname{Re}\left(\bar{\lambda} L_{22}\right)\right]_{\mu^{*}=0},
$$

where

$$
\begin{aligned}
& L_{11}=\frac{1}{4}\left(\left(\bar{f}_{1 u u}+\bar{f}_{1 v v}\right)+i\left(\bar{f}_{2 u u}+\bar{f}_{2 v v}\right)\right), \\
& L_{12}=\frac{1}{8}\left(\left(\bar{f}_{1 u u}-\bar{f}_{1 v v}+2 \bar{f}_{2 u v}\right)+i\left(\bar{f}_{2 u u}-\bar{f}_{2 v v}-2 \bar{f}_{1 u v}\right)\right), \\
& L_{21}=\frac{1}{8}\left(\left(\bar{f}_{1 u u}-\bar{f}_{1 v v}-2 \bar{f}_{2 u v}\right)+i\left(\bar{f}_{2 u u}-\bar{f}_{2 v v}+2 \bar{f}_{1 u v}\right)\right), \\
& L_{22}=\frac{1}{16}\left(\left(\bar{f}_{1 u u u}+\bar{f}_{1 u v v}+\bar{f}_{2 u u v}+\bar{f}_{2 v v v}\right)+i\left(\bar{f}_{2 u u u}+\bar{f}_{2 u v v}-\bar{f}_{2 u u v}-\bar{f}_{2 v v v}\right)\right),
\end{aligned}
$$

Keeping in mind the aforementioned computation, one has the following result.

Theorem 3. Assume that condition (55) is satisfied and $\theta \neq 0$, then the Neimark-Sacker bifurcation exists at the fixed point $\left(x_{0}, y_{0}, e_{0}\right)$ in a small neighborhood of $\mu_{2}$. Further, if $\theta<0$ (resp., $\theta>0$ ), then an attracting (resp., repelling) closed invariant curve bifurcates from the fixed point for $\mu>\mu_{2}\left(\right.$ resp., $\left.\mu<\mu_{2}\right)$.

\section{Chaos Control}

Bifurcating behavior, chaos, and unstable fluctuations have always been considered as adverse criteria in biology; therefore, these are damaging for the reproduction of the biological population. Certainly, we require to take action to stabilize the biological population. For further details and applications of chaos control methods, we refer to [37-50] and the references therein. 
In this section, we propose the following state delayed feedback control method for system (4):

$$
\left\{\begin{array}{l}
x_{n+1}=x_{n}+h x_{n}\left(d-k x_{n}-\frac{y_{n}}{a+x_{n}}-e_{n}\right)+\delta\left(x_{n}-x_{n-1}\right) \\
y_{n+1}=y_{n}+h y_{n}\left(\frac{b x_{n}}{a+x_{n}}-r\right) \\
\mu=e_{n}\left(p x_{n}-c\right)
\end{array}\right.
$$

where $\delta$ is the feedback gain for the controlled system (63).

Next, introducing $u_{n}:=x_{n}-x_{n-1}$, we obtain the following controlled system equivalent to system (63):

$$
\left\{\begin{array}{l}
x_{n+1}=x_{n}+h x_{n}\left(d-k x_{n}-\frac{y_{n}}{a+x_{n}}-e_{n}\right)+\delta u_{n} \\
y_{n+1}=y_{n}+h y_{n}\left(\frac{b x_{n}}{a+x_{n}}-r\right) \\
u_{n+1}=h x_{n}\left(d-k x_{n}-\frac{y_{n}}{a+x_{n}}-e_{n}\right)+\delta u_{n} \\
\mu=e_{n}\left(p x_{n}-c\right) .
\end{array}\right.
$$

The generalized variational matrix $J\left(x_{0}, y_{0}, u_{0}, e_{0}\right)$ of the controlled system (64) about its fixed point $\left(x_{0}, y_{0}, u_{0}, e_{0}\right)=$ $\left((\operatorname{ar} /(b-r)),\left(a+x_{0}\right)\left(d-k x_{0}-e_{0}\right), 0,\left(\mu /\left(p x_{0}-c\right)\right)\right)$ is given as follows:

$$
J\left(x_{0}, y_{0}, u_{0}, e_{0}\right)=\left(\begin{array}{cccc}
m_{11} & -\frac{h x_{0}}{a+x_{0}} & \delta & -h x_{0} \\
\frac{a b h y_{0}}{\left(a+x_{0}\right)^{2}} & 1 & 0 & 0 \\
1-m_{11} & -\frac{h x_{0}}{a+x_{0}} & \delta & -h x_{0} \\
p e_{0} & 0 & 0 & p x_{0}-c
\end{array}\right) \text {, }
$$

where

$$
m_{11}:=1+\frac{h x_{0} y_{0}}{\left(a+x_{0}\right)^{2}}-h k x_{0} .
$$

Then, generalized characteristic polynomial for $J\left(x_{0}, y_{0}\right.$, $\left.u_{0}, e_{0}\right)$ is defined as follows:

$$
F(\lambda)=\operatorname{det}\left(\begin{array}{cccc}
m_{11}-\lambda & -\frac{h x_{0}}{a+x_{0}} & \delta & -h x_{0} \\
\frac{a b h y_{0}}{\left(a+x_{0}\right)^{2}} & 1-\lambda & 0 & 0 \\
1-m_{11} & -\frac{h x_{0}}{a+x_{0}} & \delta-\lambda & -h x_{0} \\
p e_{0} & 0 & 0 & p x_{0}-c
\end{array}\right)=0,
$$

which, on simplification, yields

$$
F(\lambda)=\lambda^{3}+A \lambda^{2}+B \lambda+C,
$$

where 


$$
\begin{aligned}
& A:=\frac{p x_{0}\left(\delta+e_{0} h+m_{11}+1\right)-c\left(\delta+m_{11}+1\right)}{c-p x_{0}} \\
& B:=\frac{c\left(\left(\left(a b h^{2} x_{0} y_{0}\right) /\left(\left(a+x_{0}\right)^{3}\right)\right)+2 \delta m_{11}+m_{11}\right)+p x_{0}\left(-\left(\left(a b h^{2} x_{0} y_{0}\right) /\left(\left(a+x_{0}\right)^{3}\right)\right)-e_{0} h-2 \delta m_{11}-m_{11}\right)}{c-p x_{0}} \\
& C:=\delta-2 \delta m_{11}
\end{aligned}
$$

Keeping in view the controllability for system (63), we have the following lemma.

Lemma 2. Assume that $b>r, b c<r(a p+c),((b d-r(a k+$ $\left.d)) /\left((b-r)^{2}\right)\right)-(\mu /(r(a p+c)-b c))>0$; then the fixed point $\left(x_{0}, y_{0}, u_{0}, e_{0}\right)=\left((a r /(b-r)),\left(a+x_{0}\right)\left(d-k x_{0}-\right.\right.$ $\left.\left.e_{0}\right), 0,\left(\mu /\left(p x_{0}-c\right)\right)\right)$ of system (63) is a sink if the following conditions are satisfied:

$$
\begin{aligned}
& |A+C|<1+B \\
& |A-3 C|<3-B \\
& C^{2}+B-A C<1
\end{aligned}
$$

\section{Numerical Simulation and Discussion}

In this section, our main purpose is to validate theoretical findings with numerical simulation. For this, first of all, the existence of the period-doubling bifurcation is illustrated through particular choice of biologically feasible parametric values. Choose $a=5.8, b=8.8, c=2.1, d=3.6, k=2.5$, $r=1.6, p=2.2$, and $h=0.98$ with the variation of bifurcation parameter $\mu$ in $[0.19,0.22]$. Then, around $\mu \approx 0.211175$, system (3) undergoes period-doubling bifurcation. On the other hand, at $a=5.8, b=8.8, c=2.1$, $d=3.6, \quad k=2.5, \quad r=1.6, \quad p=2.2, \quad h=0.98, \quad$ and $\mu \equiv \mu_{1}=0.211175$, system (3) has a unique positive fixed point $(1.28889,0.642835,0.287096)$, and the characteristic polynomial for the Jacobian matrix of singular system (3) is given as follows:

$$
P(\lambda)=\lambda^{2}+0.057005 \lambda-0.942995
$$

Moreover, the roots of $P(\lambda)$ are -1 and 0.942995 . Consequently, $\left(a, b, c, d, k, p, r, \mu_{1}, h\right) \in F_{B}$, and it follows the correctness of Theorem 2. On the other hand, bifurcation diagrams for singular system (3) and corresponding largest Lyapunov exponents (LLE) are depicted in Figure 4.

Secondly, choose $a=41.5, b=85, c=2.7, d=3.4$, $k=1.4, r=3.5, p=2.3$, and $h=0.75$ with a variation of bifurcation parameter $\mu$ in $[0.4,1.1]$ for validity of Neimark-Sacker bifurcation. Then, around $\mu \approx 0.678396$, system (3) undergoes a Neimark-Sacker bifurcation. On the other hand, at $a=41.5, b=85, c=2.7, d=3.4, k=1.4$, $r=3.5, p=2.3, h=0.75$, and $\mu \equiv \mu_{2}=0.678396$, system (3) has a unique positive fixed point $(1.78221,18.1794,0.484887)$, and the characteristic polynomial for the Jacobian matrix of singular system (3) is given as follows:

$$
P(\lambda)=\lambda^{2}-1.20713 \lambda+1
$$

Moreover, the roots of $P(\lambda)$ are $0.603567-0.797313 i$ and $0.603567+0.797313 i$ with $|0.603567 \pm 0.797313 i|=1$. Consequently, $\left(a, b, c, d, k, p, r, \mu_{2}, h\right) \in H_{B}$, and it follows the correctness of Theorem 3. On the other hand, the bifurcation diagrams for singular system (3) and corresponding largest Lyapunov exponents (LLE) are depicted in Figure 5. Moreover, in the chaotic region, that is, for $\mu \in[0.678396,1.1]$, some phase portraits of system (4) are depicted in Figure 6.

Finally, in order to see the efficaciousness of delayed feedback control strategy for system (4), we choose $a=41.5$, $b=85, c=2.7, d=3.4, k=1.4, r=3.5, p=2.3, h=0.75$, and $\mu=0.95$ in the chaotic region. At these parametric values, system (4) has unique positive fixed point $\left(x_{0}, y_{0}, e_{0}\right)=(1.78221,9.77703,0.679018)$, and the complex conjugate roots of the characteristic equation for the Jacobian matrix are $0.813859 \pm 0.625908 i$ with $|0.813859 \pm 0.625908 i|=1.02671>1$. Therefore, (1.78221, $9.77703,0.679018$ ) is a source for system (4). Furthermore, for these parametric values, system (64) can be written as follows:

$$
\left\{\begin{array}{l}
x_{n+1}=x_{n}+0.75 x_{n}\left(3.4-1.4 x_{n}-\frac{y_{n}}{41.5+x_{n}}-e_{n}\right)+\delta u_{n} \\
y_{n+1}=y_{n}+0.75 y_{n}\left(\frac{85 x_{n}}{41.5+x_{n}}-3.5\right) \\
u_{n+1}=0.75 x_{n}\left(3.4-1.4 x_{n}-\frac{y_{n}}{41.5+x_{n}}-e_{n}\right)+\delta u_{n} \\
0.95=e_{n}\left(2.3 x_{n}-2.7\right) .
\end{array}\right.
$$

On the other hand, the characteristic polynomial of the Jacobian matrix of system (73) is given as follows:

$$
P(\lambda)=\lambda^{3}-(\delta+1.62772) \lambda^{2}+(2 \delta+1.05413) \lambda-\delta .
$$

Taking into account the conditions of Lemma 2, we have that the positive fixed point of system (73) is a sink if $-0.920461<\delta<-0.14539$. Consequently, system (73) is controllable in $\delta \in]-0.920461,-0.14539[$. More generally, if we take $a=41.5, b=85, c=2.7, d=3.4$, $k=1.4, r=3.5, p=2.3, h=0.75$, and $\mu \in[0.678396,1.1]$, then the controllable region for system (64) is depicted in Figure 7. 


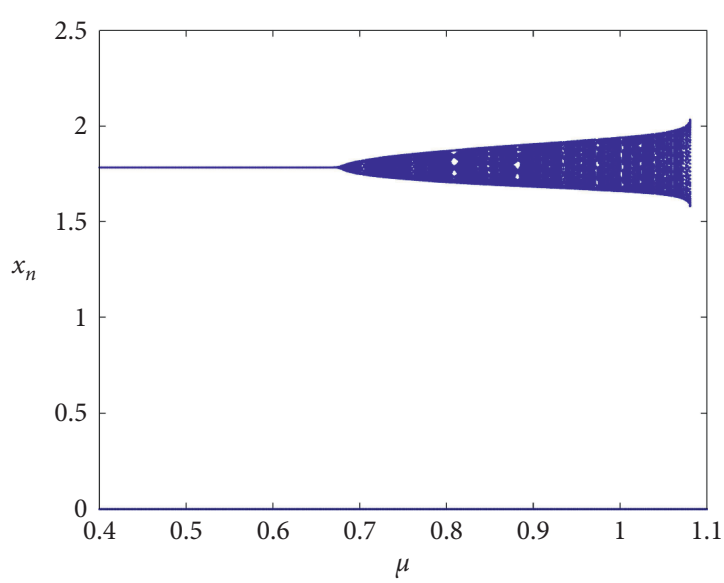

(a)

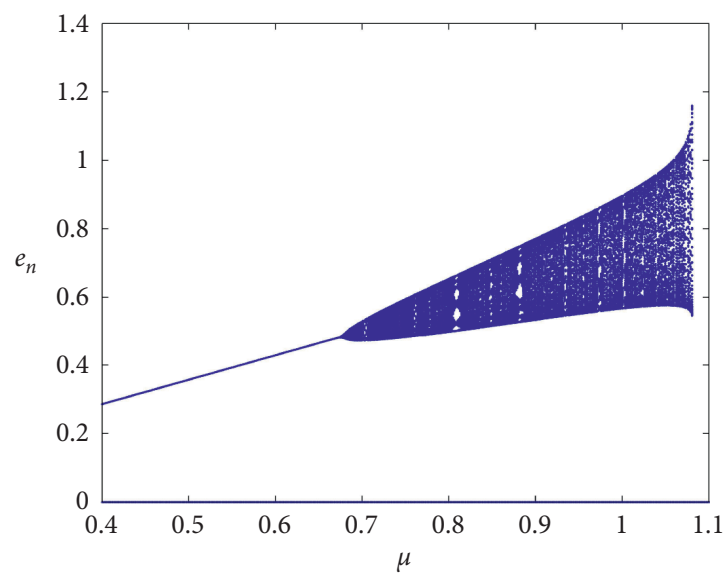

(c)

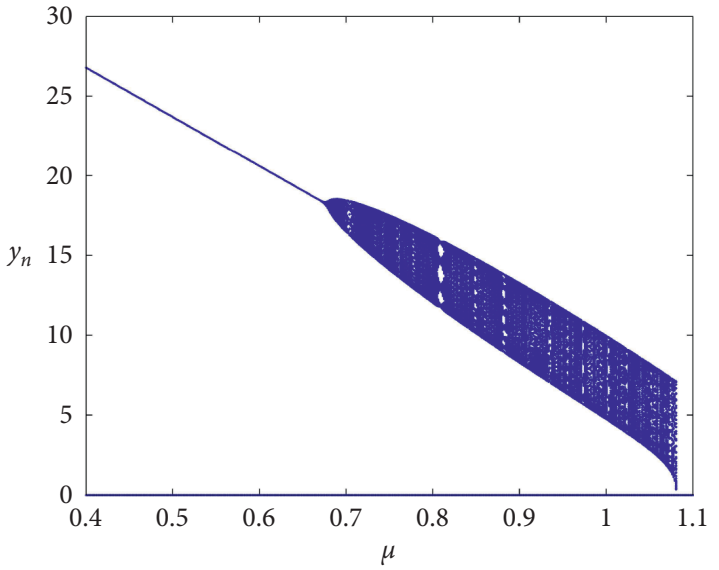

(b)

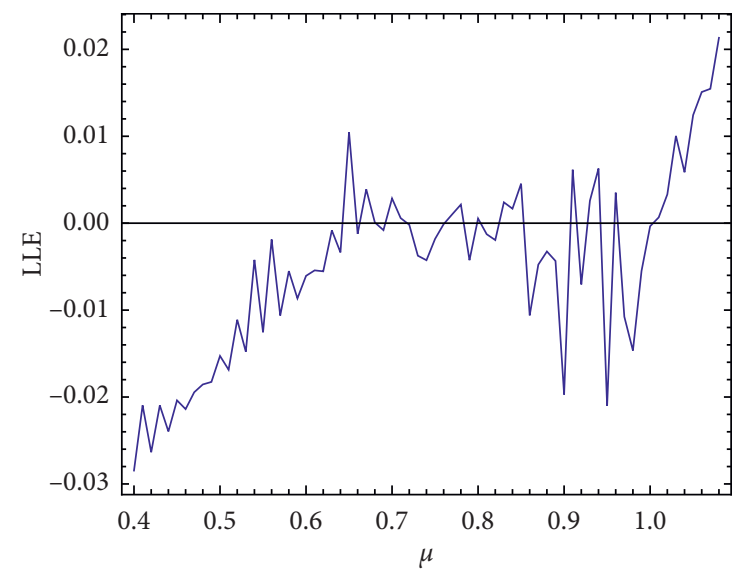

(d)

Figure 5: Bifurcation diagrams and LLE for system (3) with $a=41.5, b=85, c=2.7, d=3.4, k=1.4, r=3.5, p=2.3, h=0.75$, $\mu \in[0.4,1.1]$, and $\left(x_{0}, y_{0}, e_{0}\right)=(1.5,5.58,0.775)$. (a) Bifurcation diagram for $x_{n}$. (b) Bifurcation diagram for $y_{n}$. (c) Bifurcation diagram for $e_{n}$. (d) Largest Lyapunov exponents.

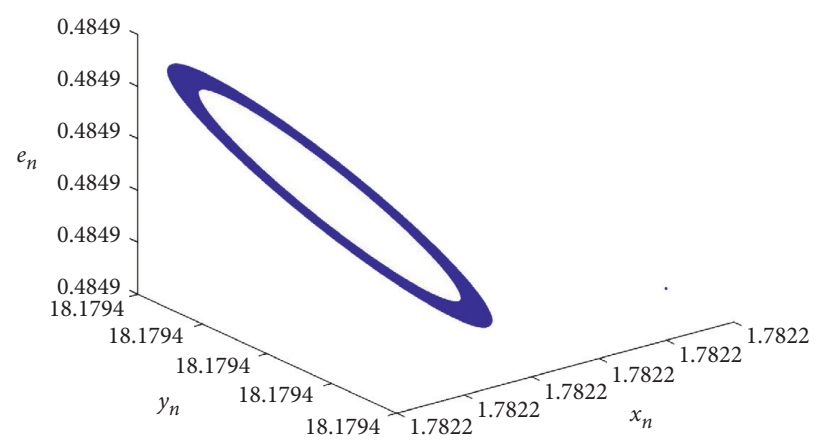

(a)

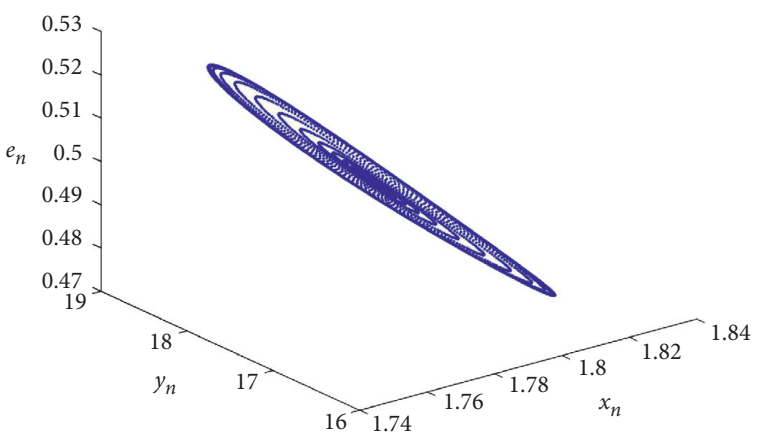

(b)

Figure 6: Continued. 


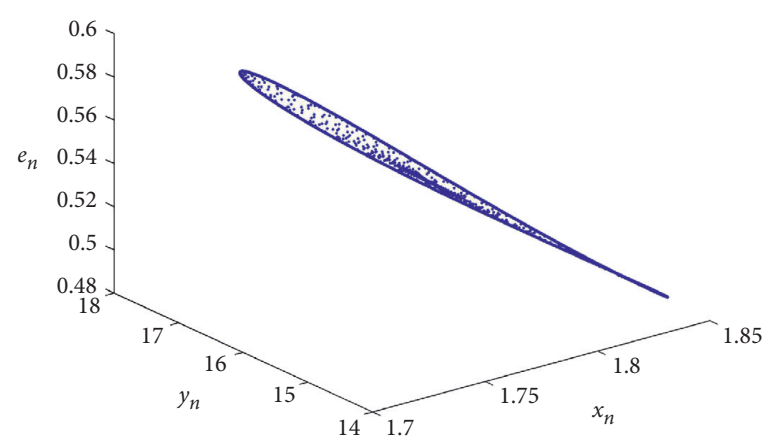

(c)

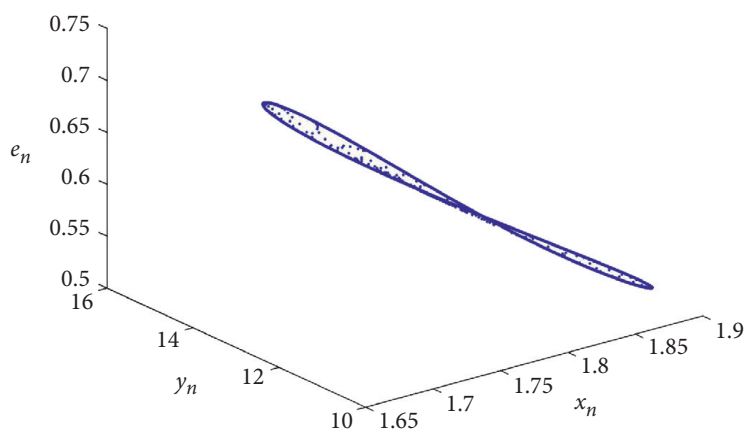

(e)

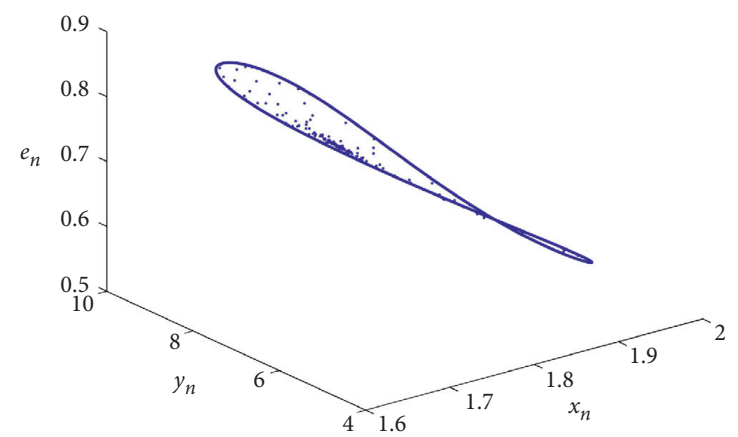

(g)

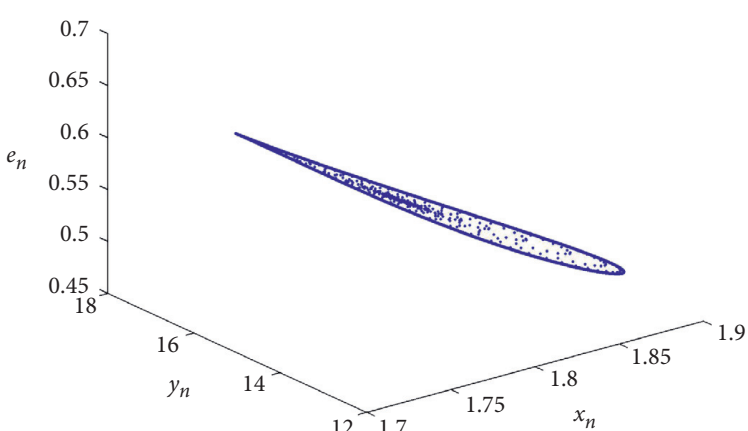

(d)

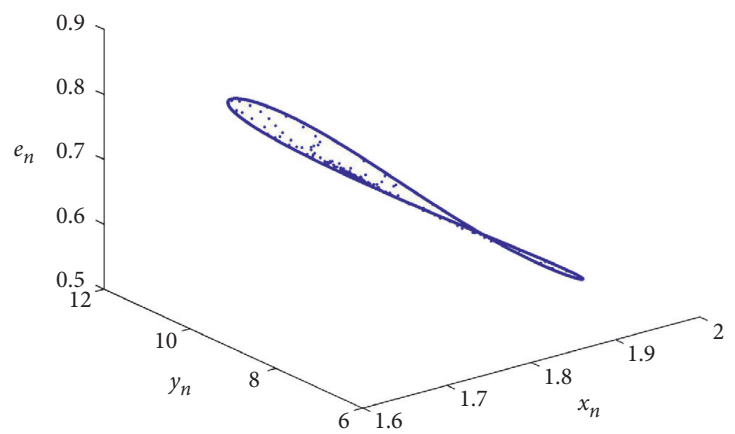

(f)

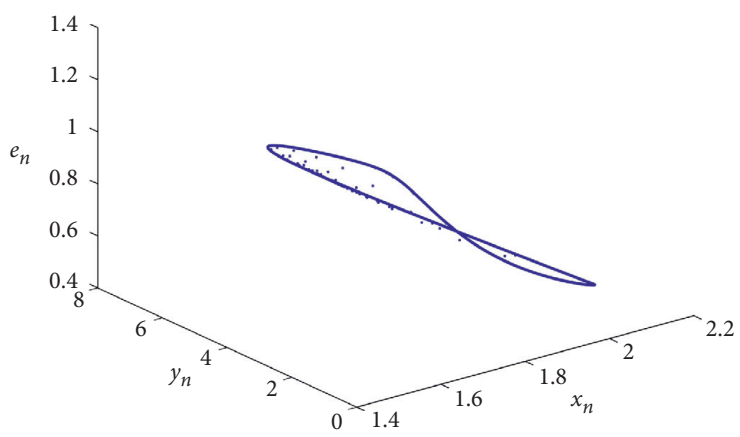

(h)

Figure 6: Phase portraits of system (4) for $a=41.5, b=85, c=2.7, d=3.4, k=1.4, r=3.5, p=2.3$, and $h=0.75$ and with different values of $\mu \in[0.678396,1.1]$. (a) Phase portrait for $\mu=0: 678396$. (b) Phase portrait for $\mu=0: 7$. (c) Phase portrait for $\mu=0: 75$. (d) Phase portrait for $\mu=0: 8$. (e) Phase portrait for $\mu=0: 85$. (f) Phase portrait for $\mu=0: 95$. (g) Phase portrait for $\mu=1$. (h) Phase portrait for $\mu=1: 08$.

\section{Conclusion}

We discuss the dynamical behavior of a discrete-time singular bioeconomic model. Moreover, Euler's approximation is implemented to a bioeconomic model governed by the differential-algebraic system proposed in [11]. The Neimark-Sacker bifurcation and period-doubling bifurcation are studied for the discrete bioeconomic model with the implementation of normal form theory, bifurcation theory, and the center manifold theorem. On the other hand, we select $\mu$ (the economic profit parameter) as a bifurcation parameter. Our investigations show richer dynamical behaviors for the discrete-time bioeconomic model compared with its continuous counterpart studied in [11]. Numerical computation for maximum Lyapunov exponents ensures further dynamical behavior and complexity of the model.
Such type of complex phenomena might result from economic profit [25]. With the variation of the bifurcation parameter $\mu$, the biologically feasible fixed point resembles the stability of economic profit, and later on, the system may sacrifice its stability by undergoing period-doubling or Neimark-Sacker bifurcation, and consequently trajectories tend to a period-doubling cascade or an invariant circle. Our theoretical discussion reveals that if the economic revenue $\mu$ increases beyond a certain threshold value $\mu_{1}$ (respectively, $\mu_{2}$ ), a phenomenon of period-doubling bifurcation (respectively, Neimark-Sacker bifurcation) occurs. From Theorem 2 (respectively, Theorem 3), if the economic revenue $\mu$ is equal to or larger than the bifurcation value $\mu_{1}$ (respectively, $\mu_{2}$ ), the predator population, the prey population, and the harvesting effort will not stay at steady states, which will result in serious biological economic 


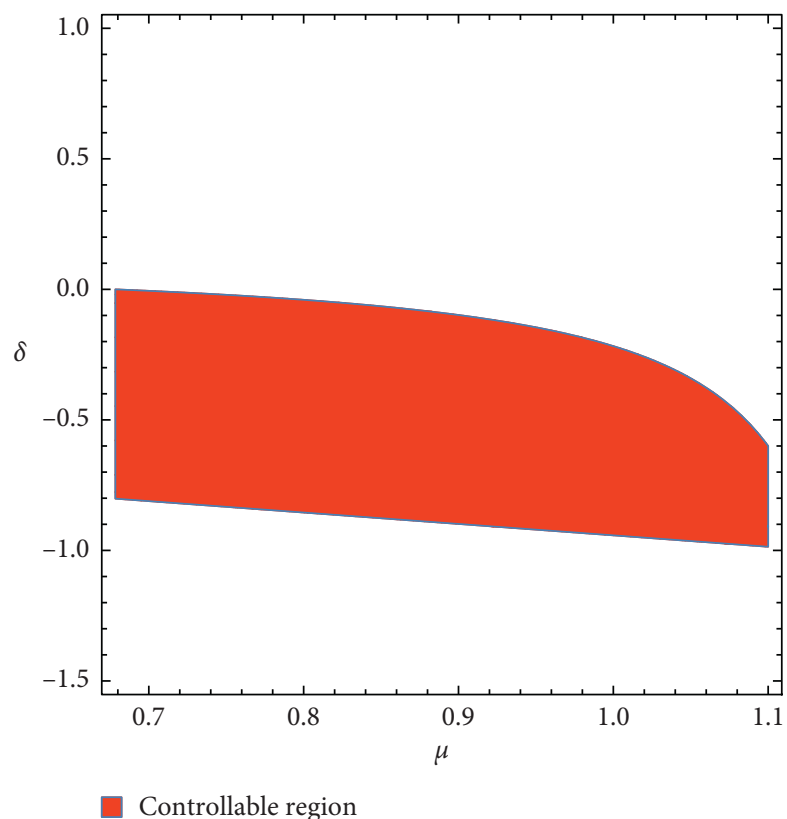

FIGURE 7: Controllability region of system (64) for $a=41.5, b=85$, $c=2.7, \quad d=3.4, \quad k=1.4, \quad r=3.5, \quad p=2.3, \quad h=0.75, \quad$ and $\mu \in[0.678396,1.1]$ in $\mu \delta$-plane.

environmental imbalance. Thus, it is sensible for fishermen to keep the economic revenue within a certain range for the purpose of maintaining the sustainable development of biological resources. On the other hand, the suggested state feedback control method of the delayed type vanishes such fluctuating phenomena and derives the bifurcating behavior of singular discrete-time economic prey-predator system towards a stable situation. Computation reveals that this delayed-type control strategy can be applied only by modifying the effort of harvesting for the prey population density by taking into account the present prey population density and the previous one. Consequently, economists can formulate some strategies to restrain or encourage the harvesting attempts in practical applications, for example, adjusting market price and revenue, abating pollution, making an allowance to fishermen, so that the biological populations can remain at their stable states.

\section{Data Availability}

No data were used to support this study.

\section{Conflicts of Interest}

The authors declare that they have no conflicts of interest.

\section{References}

[1] H. S. Gordon, "The economic theory of a common-property resource: the fishery," Journal of Political Economy, vol. 62, no. 2, pp. 124-142, 1954.

[2] M. B. Schaefer, "Some considerations of population dynamics and economics in relation to the management of the commercial marine fisheries," Journal of the Fisheries Research Board of Canada, vol. 14, no. 5, pp. 669-681, 1957.
[3] L. G. Anderson and J. C. Seijo, Bioeconomics of Fisheries Management, John Wiley \& Sons, Hoboken, NJ, USA, 2010.

[4] Y. Zhang, Q. L. Zhang, and L. C. Zhao, "Bifurcations and control in singular biological economical model with stage structure," Journal of Systems Engineering, vol. 22, no. 3, pp. 232-238, 2007.

[5] C. Liu, Q. Zhang, and X. Duan, "Dynamical behavior in a harvested differential-algebraic prey-predator model with discrete time delay and stage structure," Journal of the Franklin Institute, vol. 346, no. 10, pp. 1038-1059, 2009.

[6] K. Chakraborty, M. Chakraborty, and T. K. Kar, "Bifurcation and control of a bioeconomic model of a prey-predator system with a time delay," Nonlinear Analysis: Hybrid Systems, vol. 5, no. 4, pp. 613-625, 2011.

[7] Q. Zhang, X. Zhang, and C. Liu, "A singular bioeconomic model with diffusion and time delay," Journal of Systems Science and Complexity, vol. 24, no. 2, pp. 277-290, 2011.

[8] Q. Zhang, C. Liu, and X. Zhang, Complexity, Analysis and Control of Singular Biological Systems, Springer, London, UK, 2012.

[9] G. Zhang, Y. Shen, and B. Chen, "Hopf bifurcation of a predator-prey system with predator harvesting and two delays,” Nonlinear Dynamics, vol. 73, no. 4, pp. 2119-2131, 2013.

[10] X. Meng and Q. Zhang, "Complex dynamics in a singular delayed bioeconomic model with and without stochastic fluctuation," Discrete Dynamics in Nature and Society, vol. 2015, Article ID 302494, 15 pages, 2015.

[11] W. Liu, C. Fu, and B. Chen, "Stability and hopf bifurcation of a predator-prey biological economic system with nonlinear harvesting rate," International Journal of Nonlinear Sciences and Numerical Simulation, vol. 16, no. 6, pp. 249-258, 2015.

[12] C. Liu, L. Yu, and Q. Zhang, "Optimal harvest control in a singular prey-predator fishery model with maturation delay and gestation delay," Discrete Dynamics in Nature and Society, vol. 2016, Article ID 4398527, 9 pages, 2016.

[13] C. Liu, N. Lu, Q. Zhang, J. Li, and P. Liu, "Modeling and analysis in a prey-predator system with commercial harvesting and double time delays," Applied Mathematics and Computation, vol. 281, pp. 77-101, 2016.

[14] M. Li, B. Chen, and H. Ye, "A bioeconomic differential algebraic predator-prey model with nonlinear prey harvesting," Applied Mathematical Modelling, vol. 42, pp. 17-28, 2017.

[15] X.-Y. Meng and Y.-Q. Wu, "Bifurcation analysis in a singular Beddington-DeAngelis predator-prey model with two delays and nonlinear predator harvesting," Mathematical Biosciences and Engineering, vol. 16, no. 4, pp. 2668-2696, 2019.

[16] B. Babaei and M. Shafiee, "Analysis and behavior control of a modified singular prey-predator model," European Journal of Control, vol. 49, pp. 107-115, 2019.

[17] M. Danca, S. Codreanu, and B. Bakó, "Detailed analysis of a nonlinear prey-predator model," Journal of Biological Physics, vol. 23, no. 1, pp. 11-20, 1997.

[18] Z. Jing and J. Yang, "Bifurcation and chaos in discrete-time predator-prey system," Chaos, Solitons \& Fractals, vol. 27, no. 1, pp. 259-277, 2006.

[19] X. Liu and D. Xiao, "Complex dynamic behaviors of a discrete-time predator-prey system," Chaos, Solitons \& Fractals, vol. 32, no. 1, pp. 80-94, 2007.

[20] H. N. Agiza, E. M. Elabbasy, H. EL-Metwally, and A. A. Elsadany, "Chaotic dynamics of a discrete prey-predator model with holling type II," Nonlinear Analysis: Real World Applications, vol. 10, no. 1, pp. 116-129, 2009.

[21] Q. Din, "Complexity and chaos control in a discrete-time prey-predator model," Communications in Nonlinear Science and Numerical Simulation, vol. 49, pp. 113-134, 2017. 
[22] Q. Din, "Stability, bifurcation analysis and chaos control for a predator-prey system," Journal of Vibration and Control, vol. 25, no. 3, pp. 612-626, 2019.

[23] Z. He and X. Jiang, "Bifurcation and chaotic behaviour of a discrete-time variable-territory predator-prey model," IMA Journal of Applied Mathematics, vol. 76, no. 6, pp. 899-918, 2011.

[24] Z. He and X. Lai, "Bifurcation and chaotic behavior of a discrete-time predator-prey system," Nonlinear Analysis: Real World Applications, vol. 12, no. 1, pp. 403-417, 2011.

[25] B. Chen and J. Chen, "Bifurcation and chaotic behavior of a discrete singular biological economic system," Applied Mathematics and Computation, vol. 219, no. 5, pp. 2371-2386, 2012.

[26] X. Wu and B. Chen, "Bifurcations and stability of a discrete singular bioeconomic system," Nonlinear Dynamics, vol. 73, no. 3, pp. 1813-1828, 2013.

[27] W. Liu, D. Cai, and J. Shi, "Dynamic behaviors of a discretetime predator-prey bioeconomic system," Advances in Difference Equations, vol. 2018, no. 1, p. 133, 2018.

[28] W. F. Lucas, Modules in Applied Mathematics: Differential Equation Models, Springer, New York, NY, USA, 1983.

[29] J. Carr, Application of Center Manifold Theory, SpringerVerlag, New York, NY, USA, 1981.

[30] J. Guckenheimer and P. Holmes, Nonlinear Oscillations, Dynamical Systems, and Bifurcations of Vector Fields, Springer-Verlag, New York, NY, USA, 1983.

[31] C. Robinson, Dynamical Systems: Stability, Symbolic Dynamics and Chaos, Boca Raton, NY, USA, 1999.

[32] S. Wiggins, Introduction to Applied Nonlinear Dynamical Systems and Chaos, Springer-Verlag, New York, NY, USA, 2003.

[33] Y.-H. Wan, "Computation of the stability condition for the hopf bifurcation of diffeomorphisms on $\$ \backslash$ mathbb $\{\mathrm{R}\} 2 \$$," SIAM Journal on Applied Mathematics, vol. 34, no. 1, pp. 167-175, 1978.

[34] Y. A. Kuznetsov, Elements of Applied Bifurcation Theory, Springer-Verlag, New York, NY, USA, 1997.

[35] X. Sun and Y. Xiao, "Multiscale system for environmentallydriven infectious disease with threshold control strategy," International Journal of Bifurcation and Chaos, vol. 28, no. 5, Article ID 1850064, 2018.

[36] B. S. Chen, X. X. Liao, and Y. Q. Liu, "Normal forms and bifurcations for the difference-algebraic systems," Acta Mathematicae Applicatae Sinica, vol. 23, no. 3, pp. 429-433, 2000, In Chinese.

[37] E. Ott, C. Grebogi, and J. A. Yorke, "Controlling chaos," Physical Review Letters, vol. 64, no. 11, pp. 1196-1199, 1990.

[38] F. J. Romeiras, C. Grebogi, E. Ott, and W. P. Dayawansa, "Controlling chaotic dynamical systems," Physica D: Nonlinear Phenomena, vol. 58, no. 1-4, pp. 165-192, 1992.

[39] E. H. Abed, H. O. Wang, and R. C. Chen, "Stabilization of period doubling bifurcations and implications for control of chaos," Physica D: Nonlinear Phenomena, vol. 70, no. 1-2, pp. 154-164, 1994.

[40] K. Ogata, Modern Control Engineering, Prentice-Hall, Englewood, NJ, USA, 2nd edition, 1997.

[41] G. Chen and X. Yu, "On time-delayed feedback control of chaotic systems," IEEE Transactions on Circuits and Systems I: Fundamental Theory and Applications, vol. 46, no. 6, pp. 767-772, 1999.

[42] G. Chen, J.-Q. Fang, Y. Hong, and H. Qin, "Controlling hopf bifurcations: discrete-time systems," Discrete Dynamics in Nature and Society, vol. 5, no. 1, pp. 29-33, 2000.
[43] X. S. Luo, G. Chen, B. Hong Wang, and J. Qing Fang, "Hybrid control of period-doubling bifurcation and chaos in discrete nonlinear dynamical systems," Chaos, Solitons \& Fractals, vol. 18, no. 4, pp. 775-783, 2003.

[44] G. Wen, D. Xu, and J. Xie, "Controlling Hopf bifurcations of discrete-time systems in resonance," Chaos, Solitons \& Fractals, vol. 23, no. 5, pp. 1865-1877, 2005.

[45] X. Zhang, Q.-1. Zhang, and V. Sreeram, "Bifurcation analysis and control of a discrete harvested prey-predator system with Beddington-DeAngelis functional response," Journal of the Franklin Institute, vol. 347, no. 7, pp. 1076-1096, 2010.

[46] L.-G. Yuan and Q.-G. Yang, "Bifurcation, invariant curve and hybrid control in a discrete-time predator-prey system," Applied Mathematical Modelling, vol. 39, no. 8, pp. 23452362, 2015.

[47] Q. Din, "A novel chaos control strategy for discrete-time brusselator models," Journal of Mathematical Chemistry, vol. 56, no. 10, pp. 3045-3075, 2018.

[48] Q. Din, N. Saleem, and M. S. Shabbir, "A class of discrete predator-prey interaction with bifurcation analysis and chaos control," Mathematical Modelling of Natural Phenomena, vol. 15, p. 60, 2020.

[49] Q. Din and K. Haider, "Discretization, bifurcation analysis and chaos control for Schnakenberg model," Journal of Mathematical Chemistry, vol. 58, no. 8, pp. 1615-1649, 2020.

[50] Q. Din and M. I. Khan, "A discrete-time model for consumer-resource interaction with stability, bifurcation and chaos control," Qualitative Theory of Dynamical Systems, vol. 20, no. 2, pp. 1-35, 2021. 